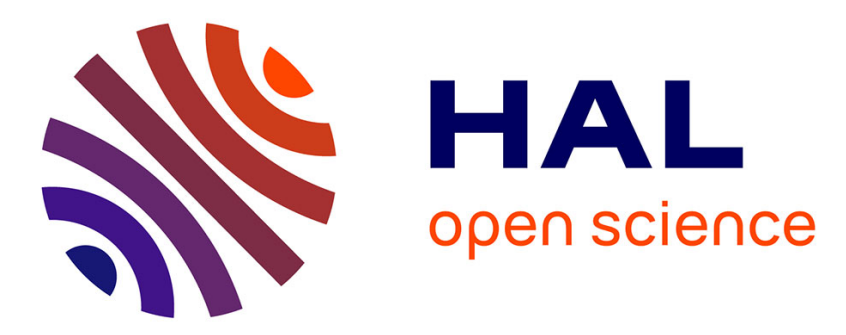

\title{
On the identification of defects in a periodic waveguide from far field data
}

\author{
Laurent Bourgeois, Sonia Fliss
}

\section{To cite this version:}

Laurent Bourgeois, Sonia Fliss. On the identification of defects in a periodic waveguide from far field data. Inverse Problems, 2014, 30 (9), 10.1088/0266-5611/30/9/095004 . hal-00914674v2

\section{HAL Id: hal-00914674 \\ https://inria.hal.science/hal-00914674v2}

Submitted on 1 Sep 2014

HAL is a multi-disciplinary open access archive for the deposit and dissemination of scientific research documents, whether they are published or not. The documents may come from teaching and research institutions in France or abroad, or from public or private research centers.
L'archive ouverte pluridisciplinaire HAL, est destinée au dépôt et à la diffusion de documents scientifiques de niveau recherche, publiés ou non, émanant des établissements d'enseignement et de recherche français ou étrangers, des laboratoires publics ou privés. 


\title{
On the identification of defects in a periodic waveguide from far field data
}

\author{
L. Bourgeois, S. Fliss \\ Laboratoire POEMS, UMR ENSTA/CNRS/INRIA, Ecole Nationale Supérieure des \\ Techniques Avancées, 32 Boulevard Victor, 75739 Paris Cedex 15, France
}

\begin{abstract}
The aim of this paper is to apply the Linear Sampling Method and the Factorization Method to retrieve some defects in a known periodic 2D waveguide from scattering data. More precisely, some far field approximations of these two sampling methods are derived. They amount to consider the so-called propagating Floquet modes as incident waves. The efficiency of the far field formulation of the LSM is shown with the help of some numerical experiments.
\end{abstract}

\section{Introduction}

The application of sampling methods $[8,17]$ to inverse scattering in homogeneous waveguides is a quite recent activity. To our best knowledge, the first contribution concerning the Linear Sampling Method in an acoustic waveguide is [22], while the first contribution concerning the Factorization Method is [9]. More recently, some contributions have enriched the literature on that field, in particular $[5,6,2,20]$ in acoustics, and $[4,7]$ in elasticity. The main issue concerning waveguides is that, any incident field being a linear combination of guided modes, only a finite number of them are propagating at long distance and hence can be used to identify some defect from far field data, the others decaying exponentially at long distance.

In particular, in [5] the authors have introduced a modal formulation of the Linear Sampling Method, that is the incident fields are guided modes instead of traditional point sources. The advantage of such modal formulation is that it enables us to define a far field formulation, which is based on the propagating guided modes only. The extension of [5] to the Factorization Method is part of [6]. In what follows we extend $[5,6]$ to the more complicated case of a periodic waveguide, by using the analysis of the forward problem contained in $[12,13]$. It is important to note that there are already a lot of contributions in the field of sampling methods in periodic gratings, both in acoustics and in electromagnetism, as can be seen in [18] and the bibliography cited therein, in particular [3]. However we insist on the fact that in these earlier works, the aim is to identify the unknown periodic geometry of 
the grating from scattering data in a homogeneous medium, while in our contribution the periodic geometry of the waveguide is known and the aim is to identify a defect within such waveguide (in other words, a loss of periodicity) from scattering data inside the periodic medium. In some sense, the case of the unknown periodic geometry looks like the case of the homogeneous waveguide, in particular the Green functions at stake are similar and have a simple form. This is in contrast with our case, for which the Green function is much more complicated (see Theorem 2.9). Potential applications of our contribution in inverse scattering for periodic waveguides concern non destructive testing (NDT) of pipelines or optical devices such as Bragg mirrors. Note, however, that the most interesting practical applications would concern NDT of biperiodic gratings in three dimensions like photonic cristals (in optics), honeycomb structures (in aeronautics) or reinforced concrete strutures (in civil engineering). The extension of our analysis of the periodic waveguides to the biperiodic structures (forward problem and inverse problem) is probably a nice and challenging avenue of research for a next future.

Our paper is organized as follows. In section 2 we describe the periodic waveguide that we consider, we recall some useful properties of the fundamental solution that are proved in $[12,13]$, and lastly we introduce the inverse problem (IP) we are interested in. In this inverse problem the incident waves are the so-called propagating Floquet modes. The section 3 is devoted to the Factorization Method in order to solve an auxiliary inverse problem (AIP) in which the incident waves are conjugated point sources, the section 4 to the Linear Sampling Method to solve another auxiliary inverse problem (AIP') in which the incident waves are point sources. From sections 3 and 4 we derive far field approximations of the Factorization Method and of the Linear Sampling Method, which both happen to solve the inverse problem (IP) of interest. In the section 6, we illustrate the efficiency of the far field approximation of the LSM on a few numerical examples. An appendix shows how the propagating Floquet modes and the fundamental solution for the homogeneous waveguide, which is a particular instance of periodic waveguides, can be deduced from the general case.

\section{Setting of the problem}

\subsection{The periodic waveguide}

Let us consider a periodic waveguide $\Omega=\mathbb{R} \times(0,1)$ of boundary $\Gamma=\partial \Omega$ in the sense that the medium that occupies $\Omega$ is characterized by a real refractive index $n_{p}(x) \in L^{\infty}(\Omega)$ which satisfies $n_{p}(x) \geq c>0$ for almost all $x \in \Omega$ and

$$
n_{p}\left(x_{1}+1, x_{2}\right)=n_{p}\left(x_{1}, x_{2}\right), \quad \forall\left(x_{1}, x_{2}\right) \in \Omega .
$$

We denote $C_{p}=(-1 / 2+p, 1 / 2+p) \times(0,1)$ for all $p \in \mathbb{Z}$, the reference cell being $C:=C_{0}=(-1 / 2,1 / 2) \times(0,1)$. For some $\xi \in(-\pi, \pi]$, a sufficiently smooth function $u$ 
is said to be $\xi$-quasiperiodic in $C_{p}$, and we note $u \in \mathrm{QP}_{\xi}\left(C_{p}\right)$, if we have

$$
u\left(1 / 2+p, x_{2}\right)=e^{i \xi} u\left(-1 / 2+p, x_{2}\right), \quad \partial_{1} u\left(1 / 2+p, x_{2}\right)=e^{i \xi} \partial_{1} u\left(-1 / 2+p, x_{2}\right) .
$$

Classically, the analysis of the periodic waveguide is based on the unbounded operator

$$
A(\xi)=-\frac{1}{n_{p}^{2}} \Delta: L^{2}\left(C, n_{p}^{2} d x_{1} d x_{2}\right) \longrightarrow L^{2}\left(C, n_{p}^{2} d x_{1} d x_{2}\right)
$$

of domain

$$
D(A(\xi))=\left\{u \in H^{1}(C), \Delta u \in L^{2}(C), \partial_{2} u=0 \quad \text { on } \quad \partial C \cap \Gamma, u \in \mathrm{QP}_{\xi}(C)\right\} .
$$

It is readily seen that the operator $A(\xi)$ is self-adjoint, positive on $L^{2}\left(C, n_{p}^{2} d x_{1} d x_{2}\right)$ and has compact resolvent. The spectrum of $A(\xi)$ is then discrete and formed by a sequence of real eigenvalues $0 \leq \lambda_{1}(\xi) \leq \lambda_{2}(\xi) \leq \ldots \leq \lambda_{n}(\xi) \leq \ldots$, with $\lim _{n \rightarrow+\infty} \lambda_{n}(\xi)=+\infty$. These eigenvalues are such that for all $n$, the function $\xi \mapsto \lambda_{n}(\xi)$ is continuous in $(-\pi, \pi]$. The corresponding eigenfunctions are denoted by $\varphi_{n}(\cdot ; \xi) \in H^{1}(C)$ and form an orthonormal basis of $L^{2}(C)$. Note that in virtue of standard regularity results for elliptic equations we have $\varphi_{n}(\cdot ; \xi) \in H^{2}(C)$ and then the functions $\varphi_{n}(\cdot ; \xi)$ are continuous up to the boundary of $C$.

As a straightforward consequence of the definition, we have (up to a correct choice of eigenfunctions)

$$
\lambda_{n}(-\xi)=\lambda_{n}(\xi), \quad \varphi_{n}(\cdot ;-\xi)=\overline{\varphi_{n}(\cdot ; \xi)} .
$$

According to [16], if the eigenvalue $\lambda_{n}\left(\xi_{0}\right)$ is simple for some $\xi_{0} \in(-\xi, \xi]$, then the functions $\xi \mapsto \lambda_{n}(\xi)$ and $\xi \mapsto \varphi_{n}(\cdot ; \xi)$ with values in $\mathbb{R}$ and $L^{2}(C)$, respectively, are analytic in a neighborhood of $\xi_{0}$.

Remark 2.1. For sake of simplicity, we have chosen to address a simple case as far as the geometry and the medium are concerned. In particular, here we consider a straight waveguide and a medium characterized by the equation $\Delta u+k^{2} n_{p}^{2} u=0$ for periodic $n_{p}$. We could easily extend the analysis which follows in the context of a waveguide with periodic geometry or a medium characterized by an equation of type $\operatorname{div}\left(\mu_{p} \nabla u\right)+\rho_{p} k^{2} u=0$ with periodic coefficients $\rho_{p}, \mu_{p}$.

\subsection{The propagating Floquet modes}

In order to define the propagating Floquet modes at frequency $k$ in a constructive way, we consider the functions $u \in H_{l o c}^{1}(\Omega):=\left\{u \in \mathcal{D}^{\prime}(\Omega), \varphi\left(x_{1}\right) u \in H^{1}(\Omega), \forall \varphi \in C_{0}^{\infty}(\mathbb{R})\right\}$ for which there exists some $\xi \in(-\pi, \pi]$ such that

$$
\left\{\begin{array}{ccc}
-\left(\Delta+k^{2} n_{p}^{2}\right) u=0 & \text { in } & \Omega \\
\partial_{\nu} u=0 & \text { on } & \Gamma \\
u \in \mathrm{QP}_{\xi}\left(C_{p}\right) & \text { for all } & p \in \mathbb{Z} .
\end{array}\right.
$$


Lemma 2.2. For fixed $\xi \in(-\pi, \pi]$, the solutions of problem (2) are defined for all $(x, p) \in C \times \mathbb{Z}$ by

$$
u\left(x_{1}+p, x_{2} ; \xi\right)=\sum_{\left\{n>0, \lambda_{n}(\xi)=k^{2}\right\}} \alpha_{n} \varphi_{n}\left(x_{1}, x_{2} ; \xi\right) e^{i p \xi},
$$

where $\alpha_{n} \in \mathbb{C}$.

Proof. If we consider the restriction to cell $C$ of a solution $u$ of system $(2)$, since the $\left(\varphi_{n}(\cdot ; \xi)\right)_{n}$ form a basis of $L^{2}(C)$, there exist some $\alpha_{n} \in \mathbb{C}$ for all $n>0$ such that $u=\sum_{n} \alpha_{n} \varphi_{n}(\cdot ; \xi)$ in $C$. Applying the operator $A(\xi)$ to such equation, we obtain

$$
\alpha_{n}\left(k^{2}-\lambda_{n}(\xi)\right) \varphi_{n}(\cdot ; \xi)=0, \quad \forall n>0,
$$

that is either $\alpha_{n}=0$ or $\lambda_{n}(\xi)=k^{2}$. The regularity of the solution $u$ implies the continuity of the trace and of the normal derivative of the function across the transverse sections of each cell $C_{p}$. Since in addition $u$ is $\xi$-quasiperiodic in each $C_{p}$, this implies that $\left.u\right|_{C_{p}}=\left.e^{i p \xi} u\right|_{C}$, and then (3).

Conversely, solutions of (3) satisfy the system (2).

Defining the sets

$$
I(k)=\left\{n>0, \exists \xi \in(-\pi, \pi], \quad \lambda_{n}(\xi)=k^{2}\right\}
$$

and for $n \in I(k)$,

$$
\Xi_{n}(k)=\left\{\xi \in(-\pi, \pi], \quad \lambda_{n}(\xi)=k^{2}\right\},
$$

we now define the propagating Floquet modes at frequency $k$, for some $(x, p) \in C \times \mathbb{Z}$, by

$$
u_{n}\left(x_{1}+p, x_{2} ; \xi\right)=\varphi_{n}\left(x_{1}, x_{2} ; \xi\right) e^{i p \xi}, \quad \forall n \in I(k), \quad \forall \xi \in \Xi_{n}(k) .
$$

It is proved in [12] that both sets $I(k)$ and $\Xi_{n}(k)$ are finite, and that $\Xi_{n}(k)$ is symmetric with respect to $\xi=0$.

Remark 2.3. In the appendix, we characterize with this approach the propagating Floquet modes in the case of the homogeneous waveguide, which is a particular case of periodic waveguides with $n_{p}=1$ in $\Omega$. In particular, we prove in that case that the above definition of the propagating Floquet modes coincide with the classical definition of the propagating guided modes.

In the remainder of the paper, we make three different assumptions on the frequency $k$. Assumption 2.4. The frequency $k>0$ is such that for all $n \in I(k)$ and all $\xi \in \Xi_{n}(k)$, $\lambda_{n}(\xi)$ is a simple eigenvalue.

Assumption 2.5. The frequency $k>0$ is such that for all $n \in I(k)$ and all $\xi \in \Xi_{n}(k)$, $\lambda_{n}^{\prime}(\xi) \neq 0$. 
Assumption 2.6. Let us consider $S_{ \pm}^{0}$ the transverse sections of the reference cell $C$ defined by $x_{1}= \pm 1 / 2$, the subdomains of $\Omega$ defined by $\Omega_{+}^{0}=\Omega \cap\left\{x_{1}>1 / 2\right\}$ and $\Omega_{-}^{0}=\Omega \cap\left\{x_{1}<-1 / 2\right\}$ and the subboundaries of $\Gamma$ defined by $\Gamma_{+}^{0}=\Gamma \cap\left\{x_{1}>1 / 2\right\}$ and $\Gamma_{-}^{0}=\Gamma \cap\left\{x_{1}<-1 / 2\right\}$.

The frequency $k$ is not an eigenvalue of the operators

of domain

$$
A_{ \pm}^{0}=-\frac{1}{n_{p}^{2}} \Delta: L^{2}\left(\Omega_{ \pm}^{0}, n_{p}^{2} d x_{1} d x_{2}\right) \longrightarrow L^{2}\left(\Omega_{ \pm}^{0}, n_{p}^{2} d x_{1} d x_{2}\right)
$$

$$
D\left(A_{ \pm}^{0}\right)=\left\{u \in H^{1}\left(\Omega_{ \pm}^{0}\right), \Delta u \in L^{2}\left(\Omega_{ \pm}^{0}\right), \partial_{2} u=0 \quad \text { on } \quad \Gamma_{ \pm}^{0}, \quad u=0 \quad \text { on } \quad S_{ \pm}^{0}\right\} .
$$

The assumption 2.4 ensures the local analytic behaviour (in particular the differentiability) of the functions $\xi \mapsto \lambda_{n}(\xi)$ and $\xi \mapsto \varphi_{n}(\cdot ; \xi)$ for a fixed $n$. The assumption 2.5 means that the functions $\xi \rightarrow \lambda_{n}(\xi)$ have no local extrema, which is necessary to apply the limiting absorption principle to scattering solutions. The assumption 2.6 means that there is no edge mode in any of the two half-waveguides associated with a homogeneous Dirichlet boundary condition, which enables us to define the Dirichlet-to-Neumann operators. The precise role of each of these assumptions will be specified in the next subsection. It is proved in [12] that the set of $k$ for which one of the assumptions 2.4, 2.5 and 2.6 does not hold is countable. It is also proved in [12] that if $n_{p}$ is symmetric with respect to the axis $x_{1}=0$, then the assumption 2.6 is automatically satisfied for any $k$. The assumption 2.4 is purely technical and enables us to give a simplified presentation of the results. By adding some technical arguments these results could be adapted if we removed assumption 2.4 (see [13]).

The group velocity of the propagating Floquet mode $u_{n}$ is given by

$$
V_{n}(\xi):=\frac{d k}{d \xi}(\xi)=\frac{1}{2} \lambda_{n}(\xi)^{-1 / 2} \lambda_{n}^{\prime}(\xi) .
$$

Thanks to the assumption 2.5, the propagating Floquet modes $u_{n}$ can be divided into those for which $\lambda_{n}^{\prime}(\xi)>0$ (they propagate from the left to the right) and those for which $\lambda_{n}^{\prime}(\xi)<0$ (they propagate from the right to the left). In the following, for $\lambda_{n}^{\prime}(\xi)>0$, we denote $u_{n}^{+}(\cdot ; \xi):=u_{n}(\cdot ; \xi)$ and $u_{n}^{-}(\cdot ; \xi):=u_{n}(\cdot ;-\xi)$. This definition is consistent with the fact that, from the first identity of $(1), \lambda_{n}^{\prime}(-\xi)=-\lambda_{n}^{\prime}(\xi)$. From the second identity of (1), it follows that

$$
u_{n}^{-}(\cdot ; \xi)=\overline{u_{n}^{+}(\cdot, \xi)}, \quad \forall n \in I(k), \quad \forall \xi \in \Xi_{n}(k), \quad \lambda_{n}^{\prime}(\xi)>0
$$

\subsection{The Green function and its far field}

Now let us consider the Green function of the periodic waveguide, denoted by $G$. Such function is defined, for all $y \in \Omega$ by the problem

$$
\left\{\begin{array}{ccc}
-\left(\Delta+k^{2} n_{p}^{2}\right) G(\cdot, y)=\delta_{y} & \text { in } & \Omega \\
\partial_{\nu} G(\cdot, y)=0 & \text { on } & \Gamma \\
G(\cdot, y) \text { satisfies RC } & \text { for } & \left|x_{1}\right| \rightarrow+\infty
\end{array}\right.
$$


The radiation condition $R C$ means that we have to select among all the solutions of the first two equations of (6) the one which is outgoing, that is the one which is "physical". A possibility to specify such radiation condition is to use the Dirichlet-to-Neumann operator, which can be properly defined with the help of the limiting absorption principle. With assumptions 2.5 and 2.6, it is proved in [12] that the problem (6) in the unbounded domain $\Omega$ is equivalent to a system in a bounded domain $\Omega_{b}=\Omega \cap\left\{x=\left(x_{1}, x_{2}\right),-1 / 2-M<\right.$ $\left.x_{1}<1 / 2+N\right\}$ for $M, N \in \mathbb{N}$, with the help of the Dirichlet-to-Neumann operators $T_{ \pm}$: $H^{\frac{1}{2}}\left(S_{ \pm}\right) \rightarrow H^{-\frac{1}{2}}\left(S_{ \pm}\right)$, where the transverse sections $S_{+}$and $S_{-}$are defined by $x_{1}=1 / 2+N$ and $x_{1}=-1 / 2-M$, respectively. Here $H^{-\frac{1}{2}}\left(S_{ \pm}\right)$is understood as the dual space of $H^{\frac{1}{2}}\left(S_{ \pm}\right)$. Such DtN operators are defined as follows. For $\varphi \in H^{\frac{1}{2}}\left(S_{+}\right)$(respectively $\left.H^{\frac{1}{2}}\left(S_{-}\right)\right), T_{+}(\varphi)$ (resp. $\left.T_{-}(\varphi)\right)$ is defined as $\left.\partial_{1} u_{+}\right|_{S_{+}} \in H^{-\frac{1}{2}}\left(S_{+}\right)$(resp. $-\left.\partial_{1} u_{-}\right|_{S_{-}} \in H^{-\frac{1}{2}}\left(S_{-}\right)$), where $u_{+}$ (resp. $u_{-}$) is the physical solution (given by the limiting absorption principle) in the halfwaveguide $\Omega_{+}=\Omega \cap\left\{x_{1}>1 / 2+N\right\}$ (resp. $\Omega_{-}=\Omega \cap\left\{x_{1}<-1 / 2-M\right\}$ ) of the problems

$$
\left\{\begin{array}{ccc}
-\left(\Delta+k^{2} n_{p}^{2}\right) u_{ \pm}=0 & \text { in } & \Omega_{ \pm} \\
\partial_{\nu} u_{ \pm}=0 & \text { on } & \Gamma_{ \pm} \\
u_{ \pm}=\varphi & \text { on } & S_{ \pm}
\end{array}\right.
$$

where $\Gamma_{+}$(resp. $\Gamma_{-}$) is the subpart of $\Gamma$ which corresponds to $x_{1}>1 / 2+N$ (resp. $\left.x_{1}<-1 / 2-M\right)$. The operators $T_{ \pm}$satisfy the following proposition, which is proved in $[12]$.

Proposition 2.7. With assumptions 2.5 and 2.6 the operators $T_{ \pm}: H^{\frac{1}{2}}\left(S_{ \pm}\right) \rightarrow H^{-\frac{1}{2}}\left(S_{ \pm}\right)$ are isomorphisms, symmetric in the sense that

$$
\forall \varphi, \psi \in H^{\frac{1}{2}}\left(S_{ \pm}\right), \quad\left\langle T_{ \pm} \varphi, \psi\right\rangle-\left\langle T_{ \pm} \psi, \varphi\right\rangle=0,
$$

and satisfy

$$
\left\langle\left(\operatorname{Im} T_{ \pm}\right) \varphi, \varphi\right\rangle \geq 0, \quad \forall \varphi \in H^{\frac{1}{2}}\left(S_{ \pm}\right),
$$

where $\langle\cdot, \cdot\rangle$ denotes duality pairing between $H^{-\frac{1}{2}}\left(S_{ \pm}\right)$and $H^{\frac{1}{2}}\left(S_{ \pm}\right)$.

Contrary to the homogeneous waveguide (see for example [5]), the operators $T_{ \pm}$do not have a simple expression in the general case of a periodic waveguide (see [12]). With the help of operators $T_{ \pm}$, assuming without loss of generality that $y \in \Omega_{b}$, the problem (6) in the unbounded domain $\Omega$ is equivalent to the following problem in the bounded domain $\Omega_{b}$ :

$$
\left\{\begin{array}{ccc}
-\left(\Delta+k^{2} n_{p}^{2}\right) G(\cdot, y)=\delta_{y} & \text { in } & \Omega_{b} \\
\partial_{\nu} G(\cdot, y)=0 & \text { on } & \Gamma_{b} \\
\partial_{\nu} G(\cdot, y)=T_{ \pm} G(\cdot, y) & \text { on } & S_{ \pm}
\end{array}\right.
$$

where $\Gamma_{b}$ is the subpart of $\Gamma$ which corresponds to $-1 / 2-M<x_{1}<1 / 2+N$.

An explicit expression of the Green function $G$ is obtained in $[12,13]$ by using the limiting absorption principle, as detailed in [13] and briefly summarized in what follows. 
Firstly one defines the Green function $G_{\varepsilon}(\cdot, y)$ in the presence of absorption $\varepsilon>0$ as the solution in $L^{2}(\Omega)$ of the system

$$
\left\{\begin{array}{ccc}
-\Delta G_{\varepsilon}(\cdot, y)-\left(k^{2}+i \varepsilon\right) n_{p}^{2} G_{\varepsilon}(\cdot, y)=\delta_{y} & \text { in } & \Omega \\
\partial_{\nu} G_{\varepsilon}(\cdot, y)=0 & \text { on } & \Gamma .
\end{array}\right.
$$

By using the Floquet-Bloch transform in the $x_{1}$ direction and the spectral decomposition of operator $A(\xi)$, one obtains that

$$
\begin{aligned}
& \forall x, y \in C, \quad \forall p, q \in \mathbb{Z} \\
& G_{\varepsilon}\left(x_{1}+p, x_{2} ; y_{1}+q, y_{2}\right)=\frac{1}{2 \pi} \sum_{n=1}^{+\infty} \int_{-\pi}^{\pi} \frac{\varphi_{n}(x ; \xi) \overline{\varphi_{n}(y ; \xi)}}{\lambda_{n}(\xi)-\left(k^{2}+i \varepsilon\right)} e^{i(p-q) \xi} d \xi .
\end{aligned}
$$

Note that the Green function $G_{\varepsilon}$ satisfies the usual symmetry relationship, that is

Lemma 2.8. For all $x, y \in \Omega$, we have $G_{\varepsilon}(x, y)=G_{\varepsilon}(y, x)$.

Proof. The proof relies on the change of variable $\xi \rightarrow-\xi$ in the integral and on the relationships (1).

Secondly, the Green function without absorption is obtained by passing to the limit $\varepsilon \rightarrow 0$ in the above expression of $G_{\varepsilon}$. From [12, 13], we obtain the following theorem.

Theorem 2.9. With assumptions 2.4 and 2.5, the solution of (6) is given by

$$
\begin{gathered}
\forall x, y \in C, \quad \forall p, q \in \mathbb{Z}, \\
G\left(x_{1}+p, x_{2} ; y_{1}+q, y_{2}\right)=\frac{1}{2 \pi} \sum_{n \notin I(k)} \int_{-\pi}^{\pi} \frac{\varphi_{n}(x ; \xi) \overline{\varphi_{n}(y ; \xi)}}{\lambda_{n}(\xi)-k^{2}} e^{i(p-q) \xi} d \xi \\
+\frac{1}{2 \pi} \sum_{n \in I(k)}\left\{\text { p.v. } \int_{-\pi}^{\pi} \frac{\varphi_{n}(x ; \xi) \overline{\varphi_{n}(y ; \xi)}}{\lambda_{n}(\xi)-k^{2}} e^{i(p-q) \xi} d \xi+i \pi \sum_{\xi \in \Xi_{n}(k)} \frac{\varphi_{n}(x ; \xi) \overline{\varphi_{n}(y ; \xi)}}{\left|\lambda_{n}^{\prime}(\xi)\right|} e^{i(p-q) \xi}\right\} .
\end{gathered}
$$

As a trivial consequence of Lemma 2.8, it follows that

Lemma 2.10. For all $x, y \in \Omega$, we have $G(x, y)=G(y, x)$.

With a view to address an inverse problem based on far field data, we will need the following result concerning the far field of the Green function, which is part of [13].

Theorem 2.11. With assumptions 2.4 and 2.5, we have

$$
\begin{aligned}
& \forall x, y \in C, \quad \forall p, q \in \mathbb{Z}, \quad \pm:=\operatorname{sgn}(p-q), \\
& G\left(x_{1}+p, x_{2} ; y_{1}+q, y_{2}\right)=G_{\infty}^{ \pm}\left(x_{1}+p, x_{2} ; y_{1}+q, y_{2}\right)+\mathcal{O}_{L^{2}}\left(e^{-\alpha|p|}\right),
\end{aligned}
$$


where

$$
G_{\infty}^{ \pm}\left(x_{1}+p, x_{2} ; y_{1}+q, y_{2}\right)=i \sum_{n \in I(k)} \sum_{\substack{\xi \in \Xi_{n}(k) \\ \pm \lambda_{n}^{\prime}(\xi)>0}} \frac{\varphi_{n}(x ; \xi) \overline{\varphi_{n}(y ; \xi)}}{\left|\lambda_{n}^{\prime}(\xi)\right|} e^{i(p-q) \xi}
$$

and where for some $\alpha>0, \mathcal{O}_{L^{2}}\left(e^{-\alpha|p|}\right)$ is for all $p$ a function the $L^{2}\left(C_{p}\right)$ norm of which is of order $e^{-\alpha|p|}$.

The proof of Theorem 2.11 relies on the one hand on some analyticity arguments to deal with the first sum (10) in the expression of $G$ and on the other hand on the non stationary phase Theorem to deal with each principal value in the sum (11) (see [13]). It follows from the definition of the propagating Floquet modes $u_{n}^{+}$and $u_{n}^{-}$that an alternative (simpler) expression of function $G_{\infty}^{ \pm}$is the following one, which will be used to solve the inverse problem in the presence of far field data.

Corollary 2.12. We have

$$
\forall x, y \in \Omega, \quad G_{\infty}^{ \pm}(x, y)=i \sum_{n \in I(k)} \sum_{\substack{\xi \in \Xi_{n}(k) \\ \lambda_{n}^{\prime}(\xi)>0}} \frac{u_{n}^{ \pm}(x ; \xi) u_{n}^{\mp}(y ; \xi)}{\lambda_{n}^{\prime}(\xi)}
$$

Proof. From Theorem 2.11, we have for $x, y \in C$ and for $p, q \in \mathbb{Z}$,

$$
G_{\infty}^{+}\left(x_{1}+p, x_{2} ; y_{1}+q, y_{2}\right)=i \sum_{n \in I(k)} \sum_{\substack{\xi \in \Xi_{n}(k) \\ \lambda_{n}^{\prime}(\xi)>0}} \frac{\varphi_{n}(x ; \xi) \overline{\varphi_{n}(y ; \xi)}}{\lambda_{n}^{\prime}(\xi)} e^{i(p-q) \xi},
$$

which by using the fact that for $\lambda_{n}^{\prime}(\xi)>0, u_{n}^{+}\left(x_{1}+p, x_{2} ; \xi\right)=\varphi_{n}(x ; \xi) e^{i p \xi}$ and the relationship (5) implies that

$$
G_{\infty}^{+}\left(x_{1}+p, x_{2} ; y_{1}+q, y_{2}\right)=i \sum_{n \in I(k)} \sum_{\substack{\xi \in \Xi_{n}(k) \\ \lambda_{n}^{\prime}(\xi)>0}} \frac{u_{n}^{+}\left(x_{1}+p, x_{2} ; \xi\right) u_{n}^{-}\left(y_{1}+q, y_{2} ; \xi\right)}{\lambda_{n}^{\prime}(\xi)} .
$$

Let us consider the other case.

$$
G_{\infty}^{-}\left(x_{1}+p, x_{2} ; y_{1}+q, y_{2}\right)=i \sum_{n \in I(k)} \sum_{\substack{\xi \in \Xi_{n}(k) \\-\lambda_{n}^{\prime}(\xi)>0}} \frac{\varphi_{n}(x ; \xi) \overline{\varphi_{n}(y ; \xi)}}{-\lambda_{n}^{\prime}(\xi)} e^{i(p-q) \xi}
$$

By using the fact that the sets $\Xi_{n}(k)$ are symmetric with respect to 0 and by using the change of variable $\xi \rightarrow-\xi$, we obtain

$$
G_{\infty}^{-}\left(x_{1}+p, x_{2} ; y_{1}+q, y_{2}\right)=i \sum_{n \in I(k)} \sum_{\substack{\xi \in \Xi_{n}(k) \\-\lambda_{n}^{\prime}(-\xi)>0}} \frac{\varphi_{n}(x ;-\xi) \overline{\varphi_{n}(y ;-\xi)}}{-\lambda_{n}^{\prime}(-\xi)} e^{-i(p-q) \xi} .
$$


By using again (1), in particular the fact that $\lambda_{n}^{\prime}(-\xi)=-\lambda_{n}^{\prime}(\xi)$, we end up with

$$
G_{\infty}^{-}\left(x_{1}+p, x_{2} ; y_{1}+q, y_{2}\right)=i \sum_{n \in I(k)} \sum_{\substack{\xi \in \Xi_{n}(k) \\ \lambda_{n}^{\prime}(\xi)>0}} \frac{\overline{\varphi_{n}(x ; \xi)} \varphi_{n}(y ; \xi)}{\lambda_{n}^{\prime}(\xi)} e^{-i(p-q) \xi}
$$

that is

$$
G_{\infty}^{-}\left(x_{1}+p, x_{2} ; y_{1}+q, y_{2}\right)=i \sum_{n \in I(k)} \sum_{\substack{\xi \in \Xi_{n}(k) \\ \lambda_{n}^{\prime}(\xi)>0}} \frac{u_{n}^{-}\left(x_{1}+p, x_{2} ; \xi\right) u_{n}^{+}\left(y_{1}+q, y_{2} ; \xi\right)}{\lambda_{n}^{\prime}(\xi)}
$$

which completes the proof.

Remark 2.13. In the appendix, we compute the far field of the fundamental solution in the special case of the homogeneous waveguide, that is $n_{p}=1$ in $\Omega$. In particular, we retrieve the expression obtained by using directly the well-known fundamental solution for the homogeneous waveguide.

\subsection{Some auxiliary forward problems and integral representation of their solutions}

In order to apply the factorization method of A. Kirsch [17], we consider two auxiliary forward problems. To ensure well-posedness of these two problems, we assume that in the cell $C, n_{p}$ has a constant value near the left transverse section and a constant value near the right transverse section, these two constants being possibly different. The precise statement of this assumption is:

Assumption 2.14. There exist $\alpha_{-}, \alpha_{+}>0$ and $\delta>0$ such that

$$
\begin{cases}\forall\left(x_{1}, x_{2}\right) \in(-1 / 2,-1 / 2+\delta) \times(0,1) & n_{p}\left(x_{1}, x_{2}\right)=\alpha_{-} \\ \forall\left(x_{1}, x_{2}\right) \in(1 / 2-\delta, 1 / 2) \times(0,1) & n_{p}\left(x_{1}, x_{2}\right)=\alpha_{+} .\end{cases}
$$

First, for a source term $f \in L^{2}(D)$ which is supported in an open domain $D$ with $\bar{D} \subset \Omega_{b}, u$ will denote the solution in $\Omega_{b}$ of the system

$$
\left\{\begin{array}{ccc}
-\left(\Delta+k^{2} n_{p}^{2}\right) u=f & \text { in } & \Omega_{b} \\
\partial_{2} u=0 & \text { on } & \Gamma_{b} \\
\pm \partial_{1} u=T_{ \pm} u & \text { on } & S_{ \pm} .
\end{array}\right.
$$

We have the following proposition.

Proposition 2.15. With assumptions 2.5, 2.6 and 2.14 the problem (13) has a unique solution in $H^{2}\left(\Omega_{b}\right)$.

Proof. It is easy to prove that an equivalent weak formulation of (13) is : find $u \in H^{1}\left(\Omega_{b}\right)$ such that

$$
a(u, v)=l(v), \quad \forall v \in H^{1}\left(\Omega_{b}\right)
$$


where the hermitian form $a$ and the antilinear form $l$ are defined by

$$
a(u, v)=\int_{\Omega_{b}}\left(\nabla u \cdot \nabla \bar{v}-k^{2} n_{p}^{2} u \bar{v}\right) d x-\left\langle T_{-} u, v\right\rangle_{S_{-}}-\left\langle T_{+} u, v\right\rangle_{S_{+}}
$$

and

$$
l(v)=\int_{\Omega_{b}} f \bar{v} d x .
$$

We first prove that the above problem is of Fredholm type in $H^{1}\left(\Omega_{b}\right)$. To see this we write $a=b+c$, where

$$
b(u, v)=\int_{\Omega_{b}}(\nabla u \cdot \nabla \bar{v}+u \bar{v}) d x-\left\langle T_{-}^{0} u, v\right\rangle_{S_{-}}-\left\langle T_{+}^{0} u, v\right\rangle_{S_{+}}
$$

and

$$
c(u, v)=-\int_{\Omega_{b}}\left(1+k^{2} n_{p}^{2}\right) u \bar{v} d x-\left\langle\left(T_{-}-T_{-}^{0}\right) u, v\right\rangle_{S_{-}}-\left\langle\left(T_{+}-T_{+}^{0}\right) u, v\right\rangle_{S_{+}},
$$

where $T_{ \pm}^{0}$ are the analogous Dirichlet-to-Neumann operators as $T_{ \pm}$for the homogeneous waveguide associated with $n_{p}=\alpha_{ \pm}$. It is easy to prove (see [6]) that for all $u \in H^{1}\left(\Omega_{b}\right)$

$$
\operatorname{Re}\left\langle T_{ \pm}^{0} u, u\right\rangle_{S_{ \pm}} \leq 0
$$

while the operators $T_{ \pm}-T_{ \pm}^{0}: H^{1 / 2}\left(S_{ \pm}\right) \rightarrow H^{-1 / 2}\left(S_{ \pm}\right)$are compact (see [12] given the assumption 2.14 on $\left.n_{p}\right)$. Then $b$ defines an isomorphism since $\operatorname{Re} b(u, u) \geq\|u\|_{H^{1}\left(\Omega_{b}\right)}^{2}$ and $c$ defines a compact operator. This proves that our problem is of Fredhlom type.

It remains to prove uniqueness, which amounts to prove that the system (13) for $f=0$ has only the trivial solution. Such result is proved in [13]. That the solution $u$ of (13) belongs to $H^{2}\left(\Omega_{b}\right)$ is a consequence of standard regularity results for elliptic problems.

We shall express the solution of problem (13) with the help of an integral representation formula.

Lemma 2.16. The solution $u$ of (13) is given in $\Omega$ by

$$
u(x)=\int_{D} G(x, y) f(y) d y, \quad \forall x \in \Omega .
$$

Proof. Let $x \in \Omega_{b}$ and $r>0$ such that $B(x, 2 r) \in \Omega_{b}$. Let us define $g_{x}$ and $\tilde{g}_{x}$ such that

$$
g_{x}(y)=\left\{\begin{array}{cl}
G(x, y) & y \in B(x, r) \\
0 & y \notin B(x, r)
\end{array}\right.
$$

and

$$
\tilde{g}_{x}(y)=g_{x}(y)-G(x, y)
$$


Now we take $\psi \in H_{0}^{2}(B(x, 2 r))$. The function $\tilde{g}_{x}$ satisfies

$$
\left\langle\left(\Delta+k^{2} n_{p}^{2}\right) \tilde{g}_{x}, \psi\right\rangle=\int_{B(x, 2 r)} \tilde{g}_{x}\left(\Delta+k^{2} n_{p}^{2}\right) \psi d y
$$

where the brackets $\langle\cdot, \cdot\rangle$ denote duality between $H^{-2}$ and $H_{0}^{2}$ (that $\tilde{g}_{x} \in L^{2}(B(x, 2 r)$ ) implies that $\left.\Delta \tilde{g}_{x} \in H^{-2}(B(x, 2 r))\right)$. It follows that

$$
\begin{aligned}
& \left\langle\left(\Delta+k^{2} n_{p}^{2}\right) \tilde{g}_{x}, \psi\right\rangle=-\int_{B(x, 2 r) \backslash B(x, r)} G(x, y)\left(\Delta+k^{2} n_{p}^{2}\right) \psi(y) d y \\
& =\int_{B(x, 2 r) \backslash B(x, r)}\left(\psi(y)\left(\Delta_{y}+k^{2} n_{p}^{2}\right) G(x, y)-G(x, y)\left(\Delta+k^{2} n_{p}^{2}\right) \psi(y)\right) d y \\
& =\int_{\partial B(x, r)}\left(\psi(y) \frac{\partial G(x, y)}{\partial \nu_{y}}-\frac{\partial \psi(y)}{\partial \nu_{y}} G(x, y)\right) d s(y)
\end{aligned}
$$

where $\nu_{y}$ is the unit normal oriented inside $B(x, r)$, by using the Green formula. As a result,

$$
<\left(\Delta+k^{2} n_{p}^{2}\right) g_{x}, \psi>=-\psi(x)+\int_{\partial B(x, r)}\left(\psi(y) \frac{\partial G(x, y)}{\partial \nu_{y}}-\frac{\partial \psi(y)}{\partial \nu_{y}} G(x, y)\right) d s(y) .
$$

Now let us choose $\psi=\theta u$ in the above relationship, for $\theta \in C_{0}^{\infty}(B(x, 2 r))$, with $\theta=1$ on $\overline{B(x, r)}$. That $\operatorname{supp}\left(g_{x}\right) \subset \overline{B(x, r)}$ implies that

$$
\begin{aligned}
& \left\langle\left(\Delta+k^{2} n_{p}^{2}\right) g_{x}, \theta u\right\rangle=\int_{B(x, 2 r)} g_{x}\left(\Delta+k^{2} n_{p}^{2}\right)(\theta u) d x \\
& =\int_{B(x, r)} g_{x}\left(\Delta+k^{2} n_{p}^{2}\right) u d x=-\int_{B(x, r)} g_{x} f d x,
\end{aligned}
$$

which implies that

$$
u(x)=\int_{\partial B(x, r)}\left(u(y) \frac{\partial G(x, y)}{\partial \nu_{y}}-\frac{\partial u(y)}{\partial \nu_{y}} G(x, y)\right) d s(y)+\int_{B(x, r)} G(x, y) f(y) d y .(14
$$

Next, we use the Green formula in the subdomain $\Omega_{b, r}:=\Omega_{b} \backslash \overline{B(x, r)}$.

$$
\begin{aligned}
\int_{\Omega_{b, r}} G(x, y) f(y) d s(y) & =\int_{\Omega_{b, r}}\left(u(y)\left(\Delta_{y}+k^{2} n_{p}^{2}\right) G(x, y)-G(x, y)\left(\Delta_{y}+k^{2} n_{p}^{2}\right) u(y)\right) d y \\
= & \int_{\partial B(x, r)}\left(u(y) \frac{\partial G(x, y)}{\partial \nu_{y}}-\frac{\partial u}{\partial \nu_{y}} G(x, y)\right) d s(y)+\int_{\Gamma_{b}}(\cdot) d s(y)+\int_{S_{-} \cup S_{+}}(\cdot) d s(y) .
\end{aligned}
$$

The integral over $\Gamma_{b}$ vanishes because of the boundary conditions satisfied by $G(\cdot, y)$ and $u$. Now we consider the integrals on $S_{ \pm}$.

$$
\int_{S_{ \pm}}\left(u(y) \frac{\partial G(x, y)}{\partial \nu_{y}}-\frac{\partial u}{\partial \nu_{y}} G(x, y)\right) d s(y)=\int_{S_{ \pm}}\left(u(y) T_{ \pm} G(x, y)-T_{ \pm} u(y) G(x, y)\right) d s(y) \text {. }
$$




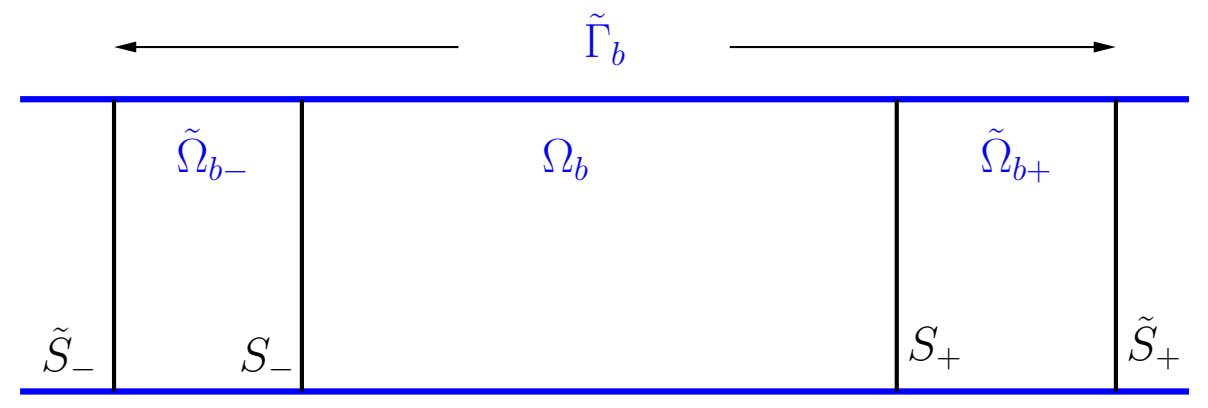

Figure 1. Notations

The above integrals vanish because the operators $T_{ \pm}$are symmetric (see Proposition 2.7). Thus we obtain that

$$
\int_{\partial B(x, r)}\left(u(y) \frac{\partial G(x, y)}{\partial \nu_{y}}-\frac{\partial u}{\partial \nu_{y}} G(x, y)\right) d s(y)=\int_{\Omega_{b} \backslash \overline{B(x, r)}} G(x, y) f(y) d s(y) \cdot(15
$$

Gathering formulas (14) and (15), we obtain the claimed result.

We now consider a second auxiliary forward problem, which is the following transmission problem : for $\left(h_{-}, h_{+}\right) \in L^{2}\left(S_{-}\right) \times L^{2}\left(S_{+}\right)$, find the solution $u$ in $\tilde{\Omega}_{b}$ of the problem

$$
\left\{\begin{array}{ccc}
-\left(\Delta+k^{2} n_{p}^{2}\right) u=0 & \text { in } & \tilde{\Omega}_{b-} \cup \Omega_{b} \cup \tilde{\Omega}_{b+} \\
\partial_{2} u=0 & \text { on } & \tilde{\Gamma}_{b} \\
{[u]_{ \pm}=0} & \text { on } & S_{ \pm} \\
{\left[\frac{\partial u}{\partial x_{1}}\right]_{ \pm}=-h_{ \pm}} & \text {on } & S_{ \pm} \\
\pm \partial_{1} u=T_{ \pm} u & \text { on } & \tilde{S}_{ \pm},
\end{array}\right.
$$

where (see notations on figure 1) $\tilde{S}_{+}$is some transverse section defined by $x_{1}=1 / 2+\tilde{N}$ with $\tilde{N}>N$ and $\tilde{S}_{-}$is some transverse section defined by $x_{1}=-1 / 2-\tilde{M}$ with $\tilde{M}>M, \tilde{\Omega}_{b}$ is the portion of $\Omega$ contained betweeen $\tilde{S}_{-}$and $\tilde{S}_{+}, \tilde{\Gamma}_{b}$ is the portion of $\Gamma$ contained betweeen $\tilde{S}_{-}$and $\tilde{S}_{+}$, we denote $\tilde{\Omega}_{b \pm}=\Omega_{ \pm} \cap \tilde{\Omega}_{b}$, and lastly $[\cdot]_{ \pm}$denote the jumps across the transverse sections $S_{ \pm}$in the direction $x_{1}$.

Proposition 2.17. With assumptions 2.5, 2.6 and 2.14 the problem (16) has a unique solution in $H^{1}\left(\tilde{\Omega}_{b}\right)$.

Proof. It is easy to prove that an equivalent weak formulation of (16) is : find $u \in H^{1}\left(\tilde{\Omega}_{b}\right)$ such that

$$
a(u, v)=l(v), \quad \forall v \in H^{1}\left(\tilde{\Omega}_{b}\right),
$$

where the hermitian form $a$ and the antilinear form $l$ are defined by

$$
a(u, v)=\int_{\tilde{\Omega}_{b}}\left(\nabla u \cdot \nabla \bar{v}-k^{2} n_{p}^{2} u \bar{v}\right) d x-\left\langle\tilde{T}_{-} u, v\right\rangle_{\tilde{S}_{-}}-\left\langle\tilde{T}_{+} u, v\right\rangle_{\tilde{S}_{+}}
$$


and

$$
l(v)=\int_{S_{-}} h_{-} \bar{v} d s+\int_{S_{+}} h_{+} \bar{v} d s .
$$

By using the same arguments as for Proposition 2.15, we prove that the above weak formulation is well-posed in $H^{1}\left(\tilde{\Omega}_{b}\right)$ as soon as assumptions 2.5, 2.6 and 2.14 are satisfied.

Lemma 2.18. The solution $u$ of problem (16) is given by

$$
u(x)=\int_{S_{-}} G(x, y) h_{-}(y) d s(y)+\int_{S_{+}} G(x, y) h_{+}(y) d s(y), \quad x \in \Omega .
$$

Proof. We first consider the case when $x \in \Omega_{b}$. By using the same technique as in the proof of Lemma 2.16, we obtain that

$$
u(x)=\int_{\partial B(x, r)}\left(u(y) \frac{\partial G(x, y)}{\partial \nu_{y}}-\frac{\partial u(y)}{\partial \nu_{y}} G(x, y)\right) d s(y)
$$

and that

$$
\begin{aligned}
& 0=\int_{\partial B(x, r)}\left(u(y) \frac{\partial G(x, y)}{\partial \nu_{y}}-\frac{\partial u}{\partial \nu_{y}} G(x, y)\right) d s(y) \\
& +\int_{S_{+}}\left(u_{-}(y) \frac{\partial G(x, y)}{\partial y_{1}}-\frac{\partial u_{-}(y)}{\partial y_{1}} G(x, y)\right) d s(y) \\
& -\int_{S_{-}}\left(u_{+}(y) \frac{\partial G(x, y)}{\partial y_{1}}-\frac{\partial u_{+}(y)}{\partial y_{1}} G(x, y)\right) d s(y),
\end{aligned}
$$

$r$ being defined as in the proof of Lemma 2.16, and with the convention that $u_{+}$(resp. $u_{-}$) is the right (resp. left) trace on a transverse section, and the same convention for the normal derivative across such section. By subtracting the two above equations we obtain

$$
\begin{aligned}
& u(x)=-\int_{S_{+}}\left(u_{-}(y) \frac{\partial G(x, y)}{\partial y_{1}}-\frac{\partial u_{-}(y)}{\partial y_{1}} G(x, y)\right) d s(y) \\
& +\int_{S_{-}}\left(u_{+}(y) \frac{\partial G(x, y)}{\partial y_{1}}-\frac{\partial u_{+}(y)}{\partial y_{1}} G(x, y)\right) d s(y) .
\end{aligned}
$$

Since by using the radiation condition of (16) and the symmetry of $T_{ \pm}$we also have

$$
\int_{S_{+}}\left(u_{+}(y) \frac{\partial G(x, y)}{\partial y_{1}}-\frac{\partial u_{+}(y)}{\partial y_{1}} G(x, y)\right) d s(y)=0
$$

and

$$
\int_{S_{-}}\left(u_{-}(y) \frac{\partial G(x, y)}{\partial y_{1}}-\frac{\partial u_{-}(y)}{\partial y_{1}} G(x, y)\right) d s(y)=0
$$


it follows that for $x \in \Omega_{b}$,

$$
\begin{aligned}
& u(x)=\int_{S_{+}}\left([u]_{+}(y) \frac{\partial G(x, y)}{\partial y_{1}}-\left[\frac{\partial u}{\partial y_{1}}\right]_{+}(y) G(x, y)\right) d s(y) \\
& +\int_{S_{-}}\left([u]_{-}(y) \frac{\partial G(x, y)}{\partial y_{1}}-\left[\frac{\partial u}{\partial y_{1}}\right]_{-}(y) G(x, y)\right) d s(y) .
\end{aligned}
$$

Using the jump relationships of (16) we arrive at

$$
u(x)=\int_{S_{+}} G(x, y) h_{+}(y) d s(y)+\int_{S_{-}} G(x, y) h_{-}(y) d s(y),
$$

which is the desired claim. The same representation formula when $x \in \tilde{\Omega}_{b-}$ or $x \in \tilde{\Omega}_{b+}$ is obtained by using the same arguments.

\subsection{The forward and inverse problems}

Now let us introduce the general forward scattering problem we consider in the following. Assume that a defect lies within the periodic waveguide, which means that in a given cell $C_{p_{0}}$, the real refractive index $n \in L^{\infty}(\Omega)$ is different from $n_{p}$ in some subdomain of $C_{p_{0}}$ and satisfies $n(x) \geq c>0$ for some constant $c>0$. More precisely, we assume that there exists an open domain $D$ of class $C^{1,1}$ such that $\bar{D}$ coincides with the support of the contrast $q=n^{2}-n_{p}^{2}$, with $\bar{D} \subset C_{p_{0}}$. For a given incident wave $u^{i}$ defined in $\Omega_{b}$, that is a smooth field solving $\Delta u^{i}+k^{2} n_{p}^{2} u^{i}=0$ in $\Omega_{b}$ and $\partial_{2} u^{i}=0$ on $\Gamma_{b}$, the scattered field $u^{s}$ is the solution in $\Omega_{b}$ of the problem

$$
\left\{\begin{array}{ccc}
-\left(\Delta+k^{2} n^{2}\right)\left(u^{s}+u^{i}\right)=0 & \text { in } & \Omega_{b} \\
\partial_{2} u^{s}=0 & \text { on } & \Gamma_{b} \\
\pm \partial_{1} u^{s}=T_{ \pm} u^{s} & \text { on } & S_{ \pm} .
\end{array}\right.
$$

We conjecture the following result.

Conjecture 2.19. The Dirichlet-to-Neumann operators $T_{ \pm}$depend analytically on $k$.

The analyticity of the DtN operators with respect to $k$, which is proved in the homogeneous case in [1], seems reasonable in the periodic case in view of the explicit (but complicated) expressions of the solutions to problems (7) given in [12]. Admitting such conjecture, we have the following theorem.

Theorem 2.20. The problem (17) has a unique solution in $H^{2}\left(\Omega_{b}\right)$ except for at most a countable set of frequencies $k$. 
Proof. In fact, the problem (17) is of Fredholm type, by using the same arguments as for Proposition 2.15. It remains to prove uniqueness except for a discrete set, this is a consequence of the Fredholm analytical Theorem as in [1], since the Dirichlet-to-Neumann operators $T_{ \pm}$depend analytically on $k$.

In the following we make the assumption:

Assumption 2.21. The frequency $k$ is such that the problem (17) is well-posed.

The inverse problem we consider in this paper is as follows.

The inverse problem (IP). Assume that we measure on $\hat{S}:=S_{-} \cup S_{+}$the scattered fields $u_{n}^{s}(\cdot, \xi)$ associated by $(17)$ with the incident fields $u^{i}$ given by the propagating Floquet modes $u_{n}(\cdot, \xi)$ for all $n \in I(k)$ and all $\xi \in \Xi_{n}(k)$. The objective is to find the support $D$ of the defect from those measurements.

\section{The factorization method}

In addition to the previous assumptions on $q \in L^{\infty}(\Omega)$, we assume that either $q(x) \geq c$ almost everywhere in $D$ or $q(x) \leq-c$ almost everywhere in $D$, for some constant $c>0$. With a view to solve the inverse problem (IP) by using the factorization method of Andreas Kirsch [17], we consider an auxiliary inverse problem.

Auxiliary inverse problem (AIP). Assume that for every point $y \in \hat{S}$, we measure on $\hat{S}$ the scattered field $\tilde{u}^{s}(\cdot, y)$ associated by (17) with the incident field $u^{i}=\overline{G(\cdot, y)}$. The objective is to find $D$ from those measurements.

In order to address such inverse problem, we now define some operators $\tilde{N}, H$ and $T$ as follows, that is the same way as in [2] for a homogeneous waveguide. The proofs of that section are very similar to those of [2]. There are however reproduced in the case of the periodic waveguide because the fundamental solution and the Dirichlet-to-Neumann operators do not have simple expressions as in [2]. In addition, considering $n_{p} \in L^{\infty}(\Omega)$ instead of $n_{p}=1$ induces less regularity for scattering solutions.

We first consider the reflectivity operator $T$, which is defined for $f \in L^{2}(D)$ by

$$
(T f)(x)=k^{2} \operatorname{sgn}(q(x))(f(x)+\sqrt{|q(x)|} v(x)), \quad x \in D,
$$

where $\operatorname{sgn}(q)=q /|q|$ and $v$ is the solution of the equation

$$
-\left(\Delta v+k^{2} n^{2} v\right)=k^{2} \frac{q}{\sqrt{|q|}} f \quad \text { in } \quad \Omega_{b}
$$

where $f$ has been extended by 0 outside $D, v$ satisfying the same boundary conditions as in problem (17). Let us remark that

$$
-\left(\Delta v+k^{2} n_{p}^{2} v\right)=\left\{\begin{array}{ccc}
\sqrt{|q|} T f & \text { in } & D \\
0 & \text { in } & \Omega_{b} \backslash \bar{D} .
\end{array}\right.
$$


We first prove that $T: L^{2}(D) \rightarrow L^{2}(D)$ is an isomorphism, which is a consequence of the two following lemmas.

Lemma 3.1. The operator $\operatorname{Re} T$ has the form $\operatorname{Re} T=T_{0}+T_{1}$ with some selfadjoint and coercive operator $T_{0}: L^{2}(D) \rightarrow L^{2}(D)$ and some compact operator $T_{1}: L^{2}(D) \rightarrow L^{2}(D)$.

Proof. With the definitions $T_{0} f:=k^{2} \operatorname{sgn}(q) f$ and $T_{1} f:=\left.k^{2} \operatorname{sgn}(q) \sqrt{|q|} v\right|_{D}$, where $v$ denotes the unique solution to equation (18) with the boundary conditions of problem (17), $T_{0}$ is clearly (positive or negative) coercive and $T_{1}$ is compact.

Lemma 3.2. The operator $T$ is injective.

Proof. Let us assume that $f \in L^{2}(D)$ and $T f=0$. If $v$ is the unique solution of equation (18) with the boundary conditions of system (17), (19) implies that $\Delta v+k^{2} n_{p}^{2} v=0$ in $\Omega_{b}$. From Lemma 2.16 it follows that $v$ vanishes in $\Omega_{b}$. Then $f$ vanishes in $D$ and hence $T$ is injective.

As an intermediate operator, we also introduce $A: L^{2}(D) \rightarrow L^{2}(\hat{S})$ which maps $f \in L^{2}(D)$ to the trace on $\hat{S}$ of the solution $v$ to equation (18) with the same boundary conditions as in problem (17). We now introduce the Herglotz operator $H$, which is defined for $h \in L^{2}(\hat{S})$ by

$$
(H h)(x)=\sqrt{|q(x)|} \int_{\hat{S}} \overline{G(x, y)} h(y) d s(y), \quad x \in D .
$$

Let us prove that $A=H^{*} T$. From (19) and Lemma 2.16 it follows that

$$
v(x)=\int_{D} \sqrt{|q(y)|} G(x, y)(T f)(y) d y, \quad \forall x \in \Omega .
$$

It remains to remark from the symmetry relationship $G(x, y)=G(y, x)$ that the formal adjoint operator of $H$ denoted by $H^{*}$ is defined, for all $f \in L^{2}(D)$, by

$$
\left(H^{*} f\right)(x)=\int_{D} \sqrt{|q(y)|} G(x, y) f(y) d s(y), \quad x \in \hat{S},
$$

which implies that $A=H^{*} T$. We hence have the mapping properties $H^{*}: L^{2}(D) \rightarrow L^{2}(\hat{S})$ and $H: L^{2}(\hat{S}) \rightarrow L^{2}(D)$. Lastly we define the near field operator $\tilde{N}$ such that for $h \in L^{2}(\hat{S})$ we have

$$
(\tilde{N} h)(x)=\int_{\hat{S}} \tilde{u}^{s}(x, y) h(y) d s(y), \quad x \in \hat{S} .
$$

Let us verify that $\tilde{N}=A H$. Indeed, for $h \in L^{2}(\hat{S})$, replacing $f$ by $H h$ in equation (18) leads to equation

$$
-\left(\Delta v+k^{2} n^{2} v\right)=k^{2} q \int_{\hat{S}} \overline{G(\cdot, y)} h(y) d s(y) .
$$


We remark that the solution $u^{s}$ of (17) solves the equation

$$
-\left(\Delta u^{s}+k^{2} n^{2} u^{s}\right)=\Delta u^{i}+k^{2} n^{2} u^{i}=k^{2} q u^{i} .
$$

Such remark leads to the fact that $v$ is the scattered field associated with the incident field $\int_{\hat{S}} \overline{G(\cdot, y)} h(y) d s(y)$, and therefore is also equal to $\int_{\hat{S}} \tilde{u}^{s}(\cdot, y) h(y) d s(y)$, and we conclude that $\tilde{N} h=A H h$. We hence have the mapping property $\tilde{N}: L^{2}(\hat{S}) \rightarrow L^{2}(\hat{S})$ as well as the following factorization decomposition for the near field operator

$$
\tilde{N}=H^{*} T H .
$$

For what follows, we will need two other lemmas concerning operators $T$ and $H$.

Lemma 3.3. The operator $\operatorname{Im} T$ satisfies $((\operatorname{Im} T) f, f)_{L^{2}(D)} \geq 0$, for all $f \in L^{2}(D)$.

Proof. We have for $f \in L^{2}(D)$,

$$
(T f, f)_{L^{2}(D)}=k^{2} \int_{D} \operatorname{sgn}(q)\left(f+\left.\sqrt{|q|} v\right|_{D}\right) \bar{f} d x
$$

where the function $v$ satisfies (18) with the boundary conditions of system (17). In particular, from (19) $v$ satisfies in $\Omega_{b}$ the equation

$$
-\left(\Delta v+k^{2} n_{p}^{2} v\right)=k^{2} \sqrt{|q|} \varphi, \quad \varphi:=\operatorname{sgn}(q)\left(f+\left.\sqrt{|q|} v\right|_{D}\right) .
$$

Then

$$
\begin{aligned}
& (T f, f)_{L^{2}(D)}=k^{2} \int_{D} \varphi(\operatorname{sgn}(q) \bar{\varphi}-\sqrt{|q|} \bar{v}) d x \\
& =k^{2} \int_{D} \operatorname{sgn}(q)|\varphi|^{2} d x+\int_{D}\left(\Delta v+k^{2} n_{p}^{2} v\right) \bar{v} d x \\
& =k^{2} \int_{D} \operatorname{sgn}(q)|\varphi|^{2} d x-\int_{D}\left(|\nabla v|^{2}-k^{2} n_{p}^{2}|v|^{2}\right) d x+\int_{\partial D} \frac{\partial v}{\partial \nu} \bar{v} d s,
\end{aligned}
$$

where $\nu$ is the outward unit normal to $D$. Then

$$
((\operatorname{Im} T) f, f)_{L^{2}(D)}=\operatorname{Im}(T f, f)_{L^{2}(D)}=\operatorname{Im} \int_{\partial D} \frac{\partial v}{\partial \nu} \bar{v} d s .
$$

By using the Green formula in $\Omega_{b} \backslash \bar{D}$ and the boundary conditions satisfied by $v$, we obtain

$$
((\operatorname{Im} T) f, f)_{L^{2}(D)}=\operatorname{Im} \int_{S_{+}}\left(T_{+} v\right) \bar{v} d s+\operatorname{Im} \int_{S_{-}}\left(T_{-} v\right) \bar{v} d s,
$$

and we complete the proof by using Proposition 2.7.

It remains to prove that

Lemma 3.4. The operator $H$ is compact and injective. 
Proof. To prove that $H$ is compact, from $A=H^{*} T$ it suffices to prove that $A$ is compact, which is true since the trace on $\hat{S}$ of a $H^{2}$ function belongs to $H^{\frac{3}{2}}(\hat{S})$. Proving the injectivity of $H$ amounts to prove the injectivity of the simpler operator $J: L^{2}(\hat{S}) \rightarrow L^{2}(D)$ such that

$$
(J h)(x)=\int_{\hat{S}} G(x, y) h(y) d s(y), \quad x \in D,
$$

since $\bar{H}=\sqrt{|q|} J$. By using Proposition 2.17 and Lemma 2.18, we obtain that the function

$$
v_{h}(x):=\int_{\hat{S}} G(x, y) h(y) d s(y), \quad x \in \Omega
$$

is the solution to problem (16). Assume that the function $v_{h}$ vanishes in $D$. Since $-\left(\Delta+k^{2} n_{p}^{2}\right) v_{h}=0$ in $\Omega_{b}$, from unique continuation it follows that $v_{h}$ vanishes in $\Omega_{b}$, in particular $\left(v_{h}\right)_{-}=0$ on $S_{+}$and $\left(v_{h}\right)_{+}=0$ on $S_{-}$. Now $v_{h}$ satisfies the jump relationships

$$
\left[v_{h}\right]_{ \pm}=0, \quad\left[\frac{\partial v_{h}}{\partial x_{1}}\right]_{ \pm}=-h_{ \pm} \quad \text { on } \quad S_{ \pm} .
$$

By using the first jump relationships of $(22)$ we obtain that $\left(v_{h}\right)_{+}=0$ on $S_{+}$and $\left(v_{h}\right)_{-}=0$ on $S_{-}$. It follows by using the Dirichlet-to-Neumann operators $T_{-}$and $T_{+}$that $v_{h}$ vanishes both in $\Omega_{-}$and $\Omega_{+}$, which means in particular that $\partial v_{h} / \partial x_{1}=0$ on both sides of $S_{-}$and on both sides of $S_{+}$. From the second jump relationships of (22), we finally obtain that $h_{ \pm}=0$, which completes the proof.

Let us now recall the following abstract theorem, which is proved in $[17,19]$.

Theorem 3.5. Let $X \subset U \subset X^{*}$ be a Gelfand triple with some Hilbert spaces $U$ and $X$ such that each embedding is dense. Furthermore, let $V$ be another Hilbert space which we identify to its dual $V^{*}$, and $\tilde{N}: V \rightarrow V, H: V \rightarrow X$ and $T: X \rightarrow X^{*}$ be linear and bounded operators with $\tilde{N}=H^{*} T H$. We make the following assumptions:

(i) $H$ is compact and injective.

(ii) $\operatorname{Re} T$ has the form $\operatorname{Re} T=T_{0}+T_{1}$ with some selfadjoint and coercive operator $T_{0}: X \rightarrow$ $X^{*}$ and some compact operator $T_{1}: X \rightarrow X^{*}$.

(iii) $\operatorname{Im} T$ is non negative on $X$, that is $\langle(\operatorname{Im} T) f, f\rangle \geq 0$, for all $f \in X$.

(iv) $T$ is injective.

Then the operator $\tilde{N}_{\sharp}=|\operatorname{Re} \tilde{N}|+|\operatorname{Im} \tilde{N}|$ is a selfadjoint, positive and compact operator and the ranges of $H^{*}: X^{*} \rightarrow V$ and $\tilde{N}_{\sharp}^{\frac{1}{2}}: V \rightarrow V$ coincide.

Remark 3.6. It results from its factorization that the operator $\tilde{N}$ is injective with dense range, which is hence also the case of the operators $\tilde{N}_{\sharp}$ and $\tilde{N}_{\sharp}^{\frac{1}{2}}$.

We will also need the following proposition that characterizes $D$ by the range of $H^{*}$.

Proposition 3.7. For $z \in \Omega, z \in D$ if and only if $\left.G(\cdot, z)\right|_{\hat{S}} \in R\left(H^{*}\right)$. 
Proof. We first assume that $z \in D$. Let us prove that $\left.G(\cdot, z)\right|_{\hat{S}} \in R\left(H^{*}\right)$. Since $D$ is a $C^{1,1}$ domain, we can find a function $v \in H^{2}(D)$ such that $v=G(\cdot, z)$ and $\partial_{\nu} v=\partial_{\nu} G(\cdot, z)$ on $\partial D$. Let us define $\varphi=-\left(\Delta v+k^{2} n_{p}^{2} v\right) / \sqrt{|q|}$. Note that $\varphi \in L^{2}(D)$ because $1 / q \in L^{\infty}(D)$. From the proof of Lemma 2.16, we have for $x \in D$

$$
\begin{aligned}
& v(x)=\int_{\partial D}\left(G(x, y) \frac{\partial v}{\partial \nu}(y)-v(y) \frac{\partial G(x, y)}{\partial \nu_{y}}\right) d s(y)-\int_{D} G(x, y)\left(\Delta v+k^{2} n_{p}^{2} v\right)(y) d y \\
& =\int_{\partial D}\left(G(x, y) \frac{\partial G(y, z)}{\partial \nu_{y}}(y)-G(y, z) \frac{\partial G(x, y)}{\partial \nu_{y}}\right) d s(y)+\int_{D} \sqrt{|q(y)|} G(x, y) \varphi(y) d y \\
& =\int_{D} \sqrt{|q(y)|} G(x, y) \varphi(y) d y .
\end{aligned}
$$

The above relationship enables us to define the function $v$ in the whole domain $\Omega$, which satisfies $-\left(\Delta v+k^{2} n_{p}^{2} v\right)=0$ outside $D$, that is the same equation as $G(\cdot, z)$, as well as the same Dirichlet and Neumann boundary conditions on $\partial D$ as $G(\cdot, z)$. From unique continuation $v$ and $G(\cdot, z)$ coincide outside $D$, in particular on $\hat{S}$, that is $\left.G(\cdot, z)\right|_{\hat{S}} \in R\left(H^{*}\right)$. We now assume that $z \notin D$ and there exists $\varphi \in L^{2}(D)$ such that $\left.G(\cdot, z)\right|_{\hat{S}}=H^{*} \varphi$ Then by unique continuation, we obtain that

$$
G(x, z)=\int_{D} \sqrt{|q(y)|} G(x, y) \varphi(y) d y, \quad \forall x \in \Omega \backslash(D \cup\{z\}) .
$$

The right-hand side is a smooth function whereas the left-hand side is singular at $z$, which is a contradiction.

From Theorem 3.5 and Proposition 3.7 we readily obtain the main theorem that justifies the factorization method based on the operator $\tilde{N}$, which depends only on the data of the auxiliary inverse problem (AIP).

Theorem 3.8. For $z \in \Omega, z \in D$ if and only if $\left.G(\cdot, z)\right|_{\hat{S}} \in R\left(\tilde{N}_{\sharp}^{\frac{1}{2}}\right)$.

Proof. It suffices to check the assumptions of Theorem 3.5 with $X=U=X^{*}=L^{2}(D)$ and $V=L^{2}(\hat{S})$. The assumptions (ii), (iii) and (iv) concerning operator $T$ correspond to Lemmas 3.1, 3.3 and 3.2, respectively. The assumption (i) corresponds to Lemma 3.4.

\section{The Linear Sampling Method}

One could argue that the type of data which is required in the Factorization Method is somehow artificial: the scattered fields $\tilde{u}^{s}(\cdot, y)$ correspond to non physical incident waves, that is the conjugated point sources $\overline{G(\cdot, y)}$ for $y \in \hat{S}$. Historically, the Linear Sampling Method was introduced before the Factorization Method and was based on incident waves formed by the point sources $G(\cdot, y)$ for $y \in \hat{S}$, which are more realistic. We hence consider the following auxiliary inverse problem. 
Auxiliary inverse problem (AIP'). Assume that for every point $y \in \hat{S}$, we measure on $\hat{S}$ the scattered field $u^{s}(\cdot, y)$ associated by (17) with the incident field $u^{i}=G(\cdot, y)$. The objective is to find $D$ from those measurements.

As far as the theoretical justification of the LSM is concerned, we do not obtain a full characterization of the inclusion as for the Factorization Method (compare Theorem 3.8 and Theorem 4.1). However, as emphasized by several sampling method users, the quality of the identification with the two methods are approximately the same in waveguides (see for example [6] for a comparison).

With a view to solve the inverse problem (IP) by using the Linear Sampling Method, let us define the near field operator $N: h \in L^{2}(\hat{S}) \rightarrow L^{2}(\hat{S})$ such that

$$
(N h)(x)=\int_{\hat{S}} u^{s}(x, y) h(y) d s(y), \quad x \in \hat{S} .
$$

We clearly have from previous section

$$
N=A \bar{H}, \quad A=H^{*} T,
$$

and then the factorization

$$
N=H^{*} T \bar{H} \text {. }
$$

Now we give the main theorem that (partially) justifies the Linear Sampling Method.

Theorem 4.1. We have for $z \in \Omega$

$$
z \notin D \text { implies that }\left.G(\cdot, z)\right|_{\hat{S}} \notin \mathrm{R}(N) \text {. }
$$

Proof. From Proposition 3.7, $z \notin D$ implies that $\left.G(\cdot, z)\right|_{\hat{S}} \notin \mathrm{R}\left(H^{*}\right)$, and from the factorization (24) that $\left.G(\cdot, z)\right|_{\hat{S}} \notin \mathrm{R}(N)$.

In order to give a characterization of the above range inclusion with the help of the Tikhonov regularization, we will need the following lemma.

Lemma 4.2. For all $x, y \in \Omega, u^{s}(x, y)=u^{s}(y, x)$.

Proof. Without loss of generality, we may assume that $x, y \in \Omega_{b}$. By definition of the scattered fields $u^{s}(\cdot, y)$, we have

$$
-\left(\Delta u^{s}(\cdot, y)+n_{p}^{2} u^{s}(\cdot, y)\right)=k^{2} q\left(G(\cdot, y)+u^{s}(\cdot, y)\right),
$$

which from Lemma 2.16 implies that

$$
u^{s}(x, y)=\int_{D} k^{2} q(z) G(x, z)\left(G(z, y)+u^{s}(z, y)\right) d z .
$$

We also have

$$
u^{s}(y, x)=\int_{D} k^{2} q(z) G(y, z)\left(G(z, x)+u^{s}(z, x)\right) d z .
$$


From the two above relationships and Lemma 2.10, it follows that

$$
u^{s}(x, y)-u^{s}(y, x)=\int_{D} k^{2}\left(q(z)\left(G(z, x) u^{s}(z, y)-G(z, y) u^{s}(z, x)\right)\right) d z .
$$

We now use the fact that

$$
-\left(\Delta u^{s}(\cdot, y)+n^{2} u^{s}(\cdot, y)\right)=k^{2} q G(\cdot, y),
$$

which implies that

$$
\begin{aligned}
& u^{s}(x, y)-u^{s}(y, x) \\
& =-\int_{D}\left(u^{s}(z, y)\left(\Delta_{z} u^{s}(z, x)+n^{2} u^{s}(z, x)\right)-u^{s}(z, x)\left(\Delta_{z} u^{s}(z, y)+n^{2} u^{s}(z, y)\right)\right) d z \\
& =-\int_{D}\left(u^{s}(z, y)\left(\Delta_{z} u^{s}(z, x)+n_{p}^{2} u^{s}(z, x)\right)-u^{s}(z, x)\left(\Delta_{z} u^{s}(z, y)+n_{p}^{2} u^{s}(z, y)\right)\right) d z \\
& =-\int_{\Omega_{b}}\left(u^{s}(z, y)\left(\Delta_{z} u^{s}(z, x)+n_{p}^{2} u^{s}(z, x)\right)-u^{s}(z, x)\left(\Delta_{z} u^{s}(z, y)+n_{p}^{2} u^{s}(z, y)\right)\right) d z \\
& =-\int_{\Omega_{b}}\left(u^{s}(z, y) \Delta_{z} u^{s}(z, x)-u^{s}(z, x) \Delta_{z} u^{s}(z, y)\right) d z \\
& =-\int_{S_{ \pm}}\left(u^{s}(z, y) \frac{\partial u^{s}(z, x)}{\partial \nu_{z}}-u^{s}(z, x) \frac{\partial u^{s}(z, y)}{\partial \nu_{z}}\right) d s(z) \\
& =-\int_{S_{ \pm}}\left(u^{s}(\cdot, y) T_{ \pm} u^{s}(\cdot, x)-u^{s}(\cdot, x) T_{ \pm} u^{s}(\cdot, y)\right) d s \\
& =0 .
\end{aligned}
$$

This last equality follows from the symmetry of operators $T_{ \pm}$(see Proposition 2.7).

We will also need the following lemma concerning the inverse transmission problem, the proof of which is omitted since it is a slight adaptation of the proof given in [10] for $q(x) \geq c>0$ in $D$ or $q(x) \leq-c$ in $D$.

Lemma 4.3. Except for a countable set of $k$, the pairs of functions $v, w \in H^{2}(D)$ solving the inverse transmission problem

$$
\left\{\begin{array}{ccc}
\left(\Delta+k^{2} n^{2}\right) w=0 & \text { in } & D \\
\left(\Delta+k^{2} n_{p}^{2}\right) v=0 & \text { in } & D \\
w=v & \text { on } & \partial D \\
\partial_{\nu} w=\partial_{\nu} v & \text { on } & \partial D
\end{array}\right.
$$

necessarily vanish in $D$.

Then we can prove the following proposition. 
Proposition 4.4. Except for at most a countable set of values of $k$, the near field operator $N$ is injective and has dense range.

Proof. To prove the injectivity of $N$ except for a discrete set of $k$, assume that the inverse transmission problem has only the trivial solution $(v, w)=(0,0)$ and $N h=0$ for some $h \in L^{2}(\hat{S})$. By the unique continuation property, we obtain that the function $v^{s}$ defined by

$$
v^{s}=\int_{\hat{S}} u^{s}(\cdot, y) h(y) d s(y)
$$

vanishes outside $D$. Since $v^{s}$ is a $H^{2}$ function, $\left.v^{s}\right|_{\partial D}=0$ and $\left.\partial_{\nu} v^{s}\right|_{\partial D}=0$. On the other hand, defining

$$
v_{h}=\int_{\hat{S}} G(\cdot, y) h(y) d s(y),
$$

$w=v_{h}+v^{s}$ solves the first equation of (25) in $D$ while $v=v_{h}$ solves the second one in $D$ and $v^{s}=w-v$ solves the two homogeneous boundary conditions on $\partial D$. The pair $(v, w)$ solves the interior transmission problem (25) and then $v_{h}=0$ in $D$ by using Lemma 4.3. Owing to the injectivity of the operator $J$ given by (21), we have that $h=0$, which completes the proof of the injectivity of $N$.

It remains to prove that $N$ has a dense range. By an easy calculation we prove from Lemma 4.2 that $N^{*}=\bar{N}$, so that the injectivity implies that $N$ has a dense range.

The range characterizations given in Theorems 3.8 and 4.1 can by expressed with the help of the Tikhonov regularization, which will be used in practice to apply the Linear Sampling Method numerically. To this aim, given an operator $F: L^{2}(\hat{S}) \rightarrow L^{2}(\hat{S})$ let us define for some parameter $\varepsilon>0$ the operator $T_{\varepsilon}(F): L^{2}(\hat{S}) \rightarrow L^{2}(\hat{S})$ by

$$
T_{\varepsilon}(F):=\left(\varepsilon I+F^{*} F\right)^{-1} F^{*},
$$

where $I$ is the identity operator on $L^{2}(\hat{S})$. We recall the following standard result concerning the Tikhonov regularization of a compact operator $F: V \rightarrow V$ which is injective and has dense range (see for example [21]): $\lim _{\varepsilon \rightarrow 0}\left\|T_{\varepsilon}(F)(f)\right\|_{V}$ exists for all $f \in V$ and

$$
f \in \mathrm{R}(F) \quad \text { iff } \quad \lim _{\varepsilon \rightarrow 0}\left\|T_{\varepsilon}(F)(f)\right\|_{V}<+\infty .
$$

Such equivalence (26) is in particular true for $F=N$ (Linear Sampling Method, see proposition 4.4) and $F=\tilde{N}_{\sharp}^{\frac{1}{2}}$ (Factorization Method, see remark 3.6).

\section{Far field approximations}

After solving the auxiliary inverse problems (AIP) and (AIP'), the objective of this section is to solve the inverse problem (IP), in which the data are the scattered fields associated with the incident fields formed by the propagating Floquet modes. As it will be shown hereafter, 
solving problem (IP) is equivalent to solve either (AIP) or (AIP') by restricting to far field data instead of full near field data.

Let us consider firstly the far field approximation of the auxiliary inverse problem (AIP'). This idea is, in view of Theorem 4.1 , to approximate $G$ by $G_{\infty}$ and $N$ by $N_{\infty}$, where $G_{\infty}$ is the far field of the fundamental solution $G$ and $N_{\infty}$ is the similar operator as $N$ given by (23) once the kernel $u^{s}(\cdot, \cdot)$ has been replaced by $u_{\infty}(\cdot, \cdot)$, where $u_{\infty}(\cdot, y)$ is the far field of the scattering solution $u^{s}(\cdot, y)$ for $y \in \hat{S}$. The expression of $G_{\infty}$ is given by (12). It remains to specify $u_{\infty}$. Recalling the expression of $G_{\infty}$, that is, for all $x, y \in \Omega$,

$$
G_{\infty}^{ \pm}(x, y)=i \sum_{n \in I(k)} \sum_{\substack{\xi \in \Xi_{n}(k) \\ \lambda_{n}^{\prime}(\xi)>0}} \frac{u_{n}^{ \pm}(x ; \xi) u_{n}^{\mp}(y ; \xi)}{\lambda_{n}^{\prime}(\xi)}
$$

by linearity it is readily seen that

$$
u_{\infty}^{ \pm}(x, y)=i \sum_{n \in I(k)} \sum_{\substack{\xi \in \Xi_{n}(k) \\ \lambda_{n}^{\prime}(\xi)>0}} \frac{u_{n}^{s \pm}(x ; \xi) u_{n}^{\mp}(y ; \xi)}{\lambda_{n}^{\prime}(\xi)},
$$

where $u_{n}^{s \pm}$ is the scattered field associated with the incident field formed by the propagating Floquet mode $u_{n}^{ \pm}$.

Let us consider secondly the far field approximation of the auxiliary inverse problem (AIP). As previously, in Theorem 3.8 we replace $G$ by $G_{\infty}$ and $\tilde{N}$ by $\tilde{N}_{\infty}$, where $\tilde{N}_{\infty}$ is the similar operator as $\tilde{N}$ given by $(20)$ once the kernel $\tilde{u}^{s}(\cdot, \cdot)$ has been replaced by $\tilde{u}_{\infty}(\cdot, \cdot)$, where $\tilde{u}_{\infty}(\cdot, y)$ is the far field of the scattering solution $\tilde{u}^{s}(\cdot, y)$ for $y \in \hat{S}$. Recalling that $\tilde{u}^{s}(\cdot, y)$ is the scattered field associated with the incident field $\overline{G(\cdot, y)}$, we first compute the far field of $\bar{G}$, that is by using (5), for all $x, y \in \Omega$,

$$
\overline{G_{\infty}^{ \pm}}(x, y)=-i \sum_{n \in I(k)} \sum_{\substack{\xi \in \Xi_{n}(k) \\ \lambda_{n}^{\prime}(\xi)>0}} \frac{u_{n}^{\mp}(x ; \xi) u_{n}^{ \pm}(y ; \xi)}{\lambda_{n}^{\prime}(\xi)}
$$

which by linearity implies

$$
\tilde{u}_{\infty}^{ \pm}(x, y)=-i \sum_{n \in I(k)} \sum_{\substack{\xi \in \Xi_{n}(k) \\ \lambda_{n}^{\prime}(\xi)>0}} \frac{u_{n}^{s \mp}(x ; \xi) u_{n}^{ \pm}(y ; \xi)}{\lambda_{n}^{\prime}(\xi)} .
$$

As can be seen from the expressions (27) and (28), in the far field approximations of the inverse problems (AIP') and (AIP), that is in the far field approximations of the Linear Sampling Method and of the Factorization Method, the set of data is formed by the scattered fields $u_{n}^{s \pm}$ associated with the propagating Floquet modes $u_{n}^{ \pm}$. This fact was already mentioned in [6] for the simpler case of the homogeneous waveguide.

In a view to test some numerical experiments with the far field approximation of the Linear Sampling Method, let us give an explicit expression of operator $N_{\infty}$. The expression of 
$\tilde{N}_{\infty}$ would be obtained the same way. The operator $N_{\infty}$ is defined, for $h=\left(h_{-}, h_{+}\right) \in S_{-} \times S_{+}$ and $x \in S_{-} \cup S_{+}$, by

$$
\left(N_{\infty} h\right)(x)=\int_{S_{-}} u_{\infty}^{+}(x, y) h_{-}(y) d s(y)+\int_{S_{+}} u_{\infty}^{-}(x, y) h_{+}(y) d s(y)
$$

which from (27) and the expression of the propagating Floquet modes $u_{n}^{ \pm}$implies that for $x \in S_{-} \cup S_{+}$,

$$
\begin{aligned}
& \left(N_{\infty} h\right)(x)=i \sum_{n \in I(k)} \sum_{\substack{\xi \in \Xi_{n}(k) \\
\lambda_{n}^{\prime}(\xi)>0}} \frac{u_{n}^{s+}(x ; \xi) e^{i M \xi}}{\lambda_{n}^{\prime}(\xi)} \int_{0}^{1} \overline{\varphi_{n}\left(-1 / 2, y_{2} ; \xi\right)} h_{-}\left(y_{2}\right) d s\left(y_{2}\right) \\
& +i \sum_{n \in I(k)} \sum_{\substack{\xi \in \Xi_{n}(k) \\
\lambda_{n}^{\prime}(\xi)>0}} \frac{u_{n}^{s-}(x ; \xi) e^{i N \xi}}{\lambda_{n}^{\prime}(\xi)} \int_{0}^{1} \varphi_{n}\left(1 / 2, y_{2} ; \xi\right) h_{+}\left(y_{2}\right) d s\left(y_{2}\right) .
\end{aligned}
$$

For convenience we make use of two complete basis $\left(\psi_{m}^{ \pm}\right)_{m>0}$ of $L^{2}(] 0,1[)$, which are not a priori assumed to be orthonormal, with

$$
\int_{0}^{1} \psi_{m}^{ \pm}\left(y_{2}\right) \psi_{m^{\prime}}^{ \pm}\left(y_{2}\right) d y_{2}=M_{m m^{\prime}}^{ \pm} .
$$

We use the decompositions

$$
\begin{aligned}
& h_{-}=\sum_{m>0} h_{m}^{-} \psi_{m}^{-}, \quad h_{+}=\sum_{m>0} h_{m}^{+} \psi_{m}^{+}, \\
& \overline{\varphi_{n}(-1 / 2, \cdot ; \xi)}=\sum_{m>0} \Phi_{n m}^{-}(\xi) \psi_{m}^{-}, \quad \varphi_{n}(1 / 2, \cdot ; \xi)=\sum_{m>0} \Phi_{n m}^{+}(\xi) \psi_{m}^{+},
\end{aligned}
$$

as well as

$$
\begin{array}{ll}
\left.u_{n}^{s+}(\cdot ; \xi)\right|_{S_{-}}=\sum_{k>0} U_{n k}^{+-}(\xi) \psi_{k}^{-}, & \left.u_{n}^{s+}(\cdot ; \xi)\right|_{S_{+}}=\sum_{k>0} U_{n k}^{++}(\xi) \psi_{k}^{+}, \\
\left.u_{n}^{s-}(\cdot ; \xi)\right|_{S_{-}}=\sum_{k>0} U_{n k}^{--}(\xi) \psi_{k}^{-}, & \left.u_{n}^{s-}(\cdot ; \xi)\right|_{S_{+}}=\sum_{k>0} U_{n k}^{-+}(\xi) \psi_{k}^{+} .
\end{array}
$$

With these notations, we obtain that

$$
\begin{aligned}
& \left.\left(N_{\infty} h\right)\right|_{S_{-}}=i \sum_{k>0}\left[\sum _ { \substack { n \in I ( k ) \\
\begin{subarray} { c } { \xi \in \Xi _ { n } ( k ) \\
\lambda _ { n } ^ { \prime } ( \xi ) > 0 } } \end{subarray} } \left(\frac{U_{n k}^{+-}(\xi) e^{i M \xi}\left(\sum_{m, m^{\prime}>0} \Phi_{n m}^{-}(\xi) M_{m m^{\prime}}^{-} h_{m^{\prime}}^{-}\right)}{\lambda_{n}^{\prime}(\xi)}\right.\right. \\
& \left.\left.+\frac{U_{n k}^{--}(\xi) e^{i N \xi}\left(\sum_{m, m^{\prime}>0} \Phi_{n m}^{+}(\xi) M_{m m^{\prime}}^{+} h_{m^{\prime}}^{+}\right)}{\lambda_{n}^{\prime}(\xi)}\right)\right] \psi_{k}^{-}
\end{aligned}
$$


and

$$
\begin{aligned}
& \left.\left(N_{\infty} h\right)\right|_{S_{+}}=i \sum_{k>0}\left[\sum _ { n \in I ( k ) } \sum _ { \substack { \xi \in \Xi _ { n } ( k ) \\
\lambda _ { n } ^ { \prime } ( \xi ) > 0 } } \left(\frac{U_{n k}^{++}(\xi) e^{i M \xi}\left(\sum_{m, m^{\prime}>0} \Phi_{n m}^{-}(\xi) M_{m m^{\prime}}^{-} h_{m^{\prime}}^{-}\right)}{\lambda_{n}^{\prime}(\xi)}\right.\right. \\
& \left.\left.+\frac{U_{n k}^{-+}(\xi) e^{i N \xi}\left(\sum_{m, m^{\prime}>0} \Phi_{n m}^{+}(\xi) M_{m m^{\prime}}^{+} h_{m^{\prime}}^{+}\right)}{\lambda_{n}^{\prime}(\xi)}\right)\right] \psi_{k}^{+} .
\end{aligned}
$$

Similarly, we derive explicit expressions for $\left.G_{\infty}(\cdot, z)\right|_{S_{-}}$and $\left.G_{\infty}(\cdot, z)\right|_{S_{+}}$with the help of the basis $\left(\psi_{m}^{ \pm}\right)_{m>0}$, precisely

$$
\left.G_{\infty}(\cdot, z)\right|_{S_{-}}=i \sum_{k>0}\left[\sum_{n \in I(k)} \sum_{\substack{\xi \in \Xi_{n}(k) \\ \lambda_{n}^{\prime}(\xi)>0}}\left(\frac{e^{i\left(M+q_{z}\right) \xi} \varphi_{n}\left(z_{1}, z_{2} ; \xi\right) \Phi_{n k}^{-}(\xi)}{\lambda_{n}^{\prime}(\xi)}\right)\right] \psi_{k}^{-}
$$

and

$$
\left.G_{\infty}(\cdot, z)\right|_{S_{+}}=i \sum_{k>0}\left[\sum_{n \in I(k)} \sum_{\substack{\xi \in \Xi_{n}(k) \\ \lambda_{n}^{\prime}(\xi)>0}}\left(\frac{e^{i\left(N-q_{z}\right) \xi} \overline{\varphi_{n}\left(z_{1}, z_{2} ; \xi\right)} \Phi_{n k}^{+}(\xi)}{\lambda_{n}^{\prime}(\xi)}\right)\right] \psi_{k}^{+},
$$

where $z=\left(z_{1}+q_{z}, z_{2}\right)$ with $\left(z_{1}, z_{2}\right) \in C$ and $q_{z} \in \mathbb{Z}$.

\section{Numerical experiments with the Linear Sampling Method}

The artificial data are obtained by solving the forward problem (17) in the periodic waveguide by using the technique introduced in $[12,15]$. Broadly speaking, such technique consists of two steps. The first one consists in computing the Dirichlet-to-Neumann operators. Such operators depend on an intermediate operator which is obtained by solving a stationary Riccati equation, the coefficients of which are operators computed by using the solutions of a family of cell problems. Besides, another output of the first step is the computation of the propagating Floquet modes as well as their group velocity (see [15]). They are hence obtained by solving a complicated program, while in the homogeneous case the propagating modes and their group velocity have explicit and simple expressions. The second step consists in solving a weak formulation in a bounded domain with the help of a finite element method by using the Dirichlet-to-Neumann operators. Note that in the forward computations we have taken $p_{0}=0$, that is the defect is contained in the cell $C$. However, this is in no way an assumption that we use to solve the inverse problem (IP). In other words, to solve the inverse problem (IP) we do not assume that the index of the cell that contains the defect is a priori known. The transverse sections $S_{-}$and $S_{+}$, which are the support of the scattering data, are not symmetric with respect to $C$ and are far away from the defect. They are located at $x_{1}=-1 / 2-M$ and $x_{1}=1 / 2+N$, respectively, with $M=20$ and $N=15$. 
We solve the far field approximation of the Linear Sampling Method by using a Tikhonov regularization associated to equation

$$
N_{\infty} h=\left.G_{\infty}(\cdot, z)\right|_{\hat{S}}
$$

for all $z$ describing some sampling grid. Both members of such equation have been given explicitly with the help of transverse basis of $S_{+}$and $S_{-}$at the end of previous section. The support of the defect is then obtained in practice by plotting in the sampling grid the function $1 /\|h(z)\|_{L^{2}(\hat{S})}$, which is supposed to vanish outside such support according to Theorem 4.1. In order to reduce the number of computations, the sampling grid covers only 5 cells, precisely the cells $C_{p}$ for $p=-2,-1,0,1,2$. The periodic waveguide that we consider is characterized in $C$ by a refractive index $n_{p}=1$ everywhere except in a ball centered at $(0,0.5)$ and of radius 0.1 , in which $n_{p}=\sqrt{2}$. Several defects are considered in this numerical section (see figure 2, we recall that the contrast is defined by $q=n^{2}-n_{p}^{2}$ ).

(i) The support of $q$ is the ball centered at $(-0.2,0.8)$ and of radius 0.1 , in which $n=\sqrt{2}$ $(q=1)$.

(ii) The support of $q$ is the ball centered at $(-0.2,0.8)$ and of radius 0.1 , in which $n=2$ $(q=3)$.

(iii) The support of $q$ is the ball centered at $(0,0.5)$ and of radius 0.1 , in which $n=1$ $(q=-1)$.

(iv) The support of $q$ is the ball centered at $(0,0.5)$ and of radius 0.1 , in which $n=\sqrt{5}$ $(q=3)$.

(v) The support of $q$ is the union of the ball centered at $(-0.2,0.8)$ and of radius 0.1 , in which $n=2(q=3)$, and of the ball centered at $(0,0.5)$ and of radius 0.1 , in which $n=1(q=-1)$.

In order to introduce artificial noisy data, the fields $\left.u_{n}^{s \pm}(\cdot, \xi)\right|_{S_{-}}$and $\left.u_{n}^{s \pm}(\cdot, \xi)\right|_{S_{+}}$that are obtained by the finite element computation are contaminated by some noise of amplitude $\delta$. More precisely, we apply to each data $\hat{u}=\left.u_{n}^{s \pm}(\cdot, \xi)\right|_{\hat{S}}$ a pointwise Gaussian noise which is calibrated in order to obtain some noisy data $\hat{u}^{\delta}$ satisfying

$$
\left\|\hat{u}^{\delta}-\hat{u}\right\|_{L^{2}(\hat{S})}=\delta=\sigma\|\hat{u}\|_{L^{2}(\hat{S})},
$$

where the relative amplitude of noise $\sigma$ is prescribed. In the Tikhonov regularization, the parameter $\varepsilon$ is chosen as a function of $\delta$ following the Morozov's strategy introduced in [11], where the Linear Sampling Method in free space was considered. We use exactly the same Morozov's technique as in [5] to compute $\varepsilon$.

In what follows we study the impact of several parameters on the efficiency of the Linear Sampling Method. 

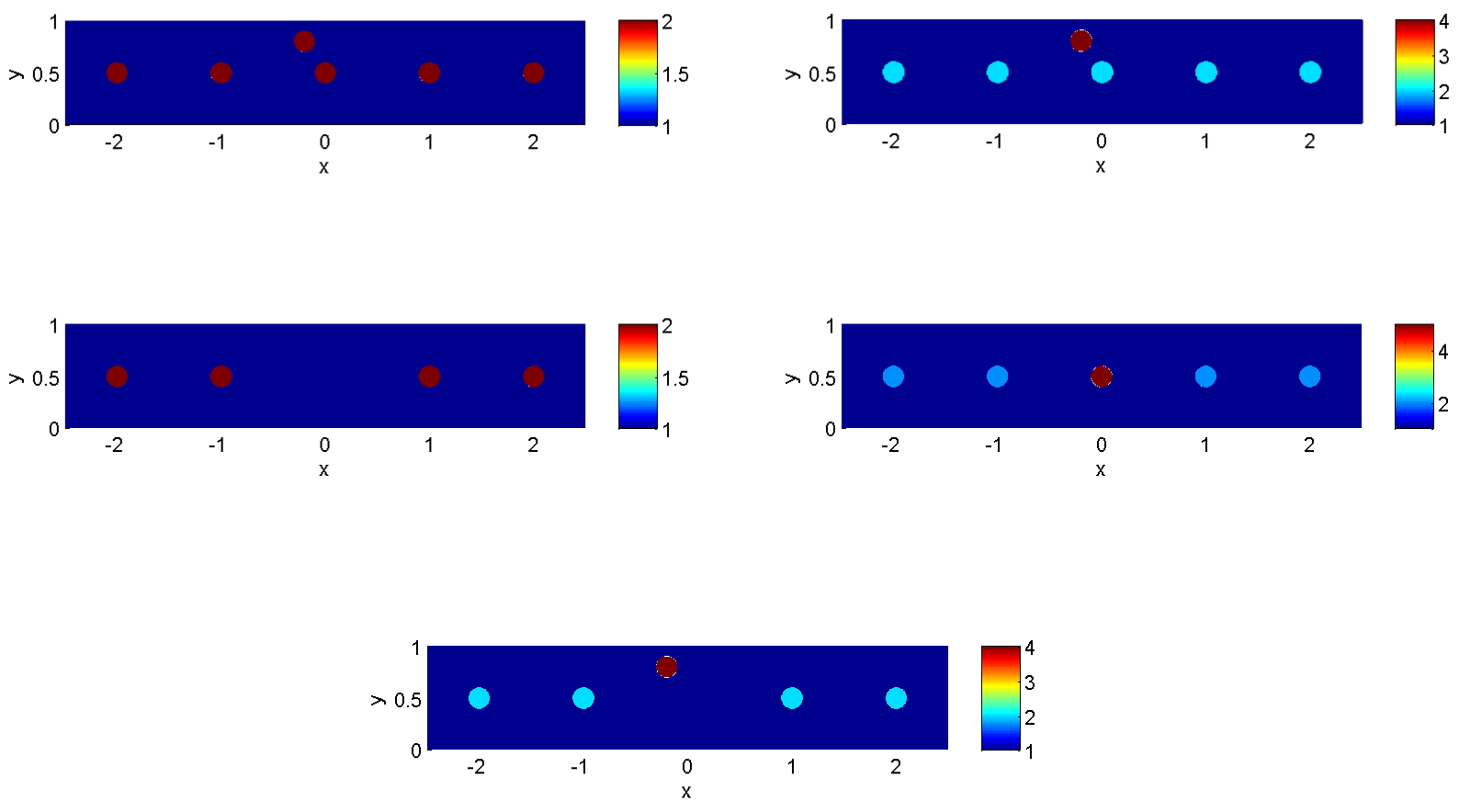

Figure 2. Level sets of $n^{2}$. Top left: defect $(i)$. Top right: defect $(i i)$. Middle left: defect (iii). Middle right: defect $(i v)$. Bottom: defect $(v)$.

\subsection{The choice of the projection basis}

Any complete basis $\left(\psi_{m}^{ \pm}\right)_{m>0}$ of $L^{2}(] 0,1[)$ can be used to project the far field approximation of the Linear Sampling Method (29) on the transverse sections $S_{ \pm}$. For numerical purposes, we have to restrict to a finite number of functions $\psi_{m}^{ \pm}$, this number is chosen equal to the number $P$ of propagating Floquet modes in each direction of propagation. Then the equation (29) amounts to solve a finite dimensional square system, the matrix to invert being a four blocks matrix (see the end of previous section). We have tried two different finite families. The first one is the orthonormal family formed by the $P$ first eigenfunctions of the $1 D$ operator $-\Delta$ in the transverse section with Neumann boundary conditions, that is

$$
\psi_{m}^{-}=\psi_{m}^{+}=\theta_{m},
$$

where the $\theta_{m}$ are given by (33). Such family is the natural family to be used for the homogeneous waveguide, like in [5]. The second one is formed by the traces of the propagating Floquet modes (4) on both sides of the reference cell $C$, precisely $\psi_{m}^{-}$is the trace on the left side of $C$ of the propagating Floquet modes which propagate to the left, while $\psi_{m}^{+}$is the 
trace on the right side of $C$ of those which propagate to the right. We hence have

$$
\psi_{m}^{-}=\overline{\varphi_{n}(-1 / 2, \cdot ; \xi)}, \quad \psi_{m}^{+}=\varphi_{n}(1 / 2, \cdot ; \xi),
$$

where the index $m$ counts the different pairs $(n, \xi)$ for $n \in I(k)$ and $\xi \in \Xi_{n}(k)$. Such family is linearly independent, which can be deduced from [14], but is not orthonormal. These two families have been tested for all the defects above. In all cases, it happens that the second family (the one based on the Floquet modes) is the best, as can be seen on the figure 3 for the defect $(i)$ and on the figure 4 for the defect $(i i i)$, when the number of propagating Floquet modes in each direction of propagation is $P=12$ and the relative amplitude of noise is $\sigma=1 \%$. From now on, the second family is used in our numerical experiments.
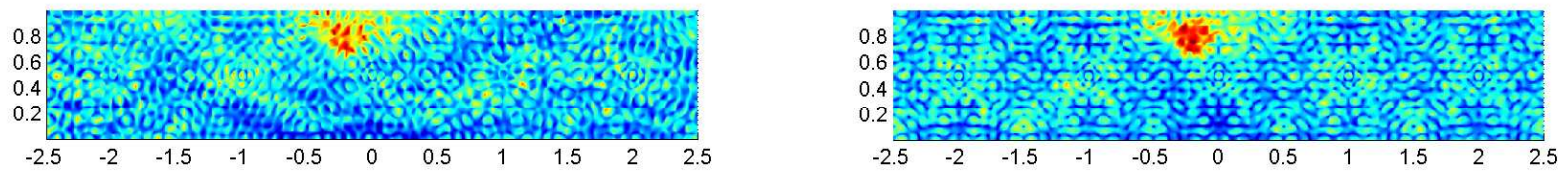

Figure 3. Defect $(i)$. Left: projection on the $\theta_{m}$. Right: projection on the traces of Floquet modes
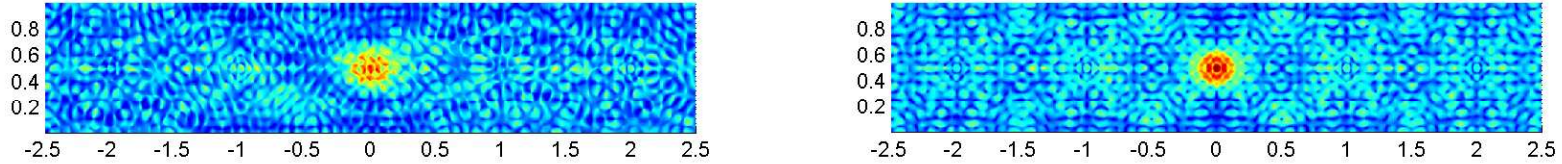

Figure 4. Defect $($ iii $)$. Left: projection on the $\theta_{m}$. Right: projection on the traces of Floquet modes

\subsection{The influence of the contrast}

We analyze the impact of the contrast on the quality of the identification. We have $P=12$ and $\sigma=1 \%$. The supports of defects $(i i)$ and $(i v)$ are the same as those of $(i)$ and (iii), respectively, but the absolute value of the contrast is higher. The results are shown on figure 5, and have to be compared to the right picture of figures 3 and 4, respectively. We note a slight degradation of the identification for higher contrast. Note that in the previous examples the sign of the contrast was constant. The case of defect $(v)$, for which our assumption on $q$ is not satisfied since the sign of $q$ is changing, is presented on figure 6 . The identification is not satisfactory in this case. 

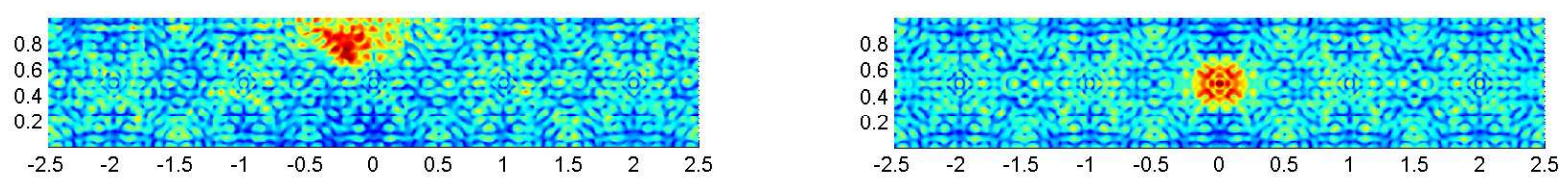

Figure 5. Left: $\operatorname{defect}(i i)$. Right: defect $(i v)$

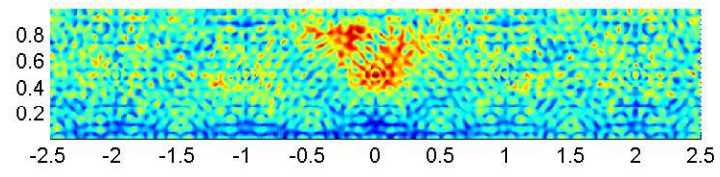

Figure 6. Defect $(v)$

\subsection{The influence of noise}

Sampling methods are well known to be robust with respect to Gaussian noise, which can be verified again in the case of the periodic waveguide. For the defect $(i)$ with $P=12$, we compare the quality of the results for different amplitudes of noise, namely $\sigma=1 \%, \sigma=5 \%$ and $\sigma=10 \%$, which correspond to figure 7 .
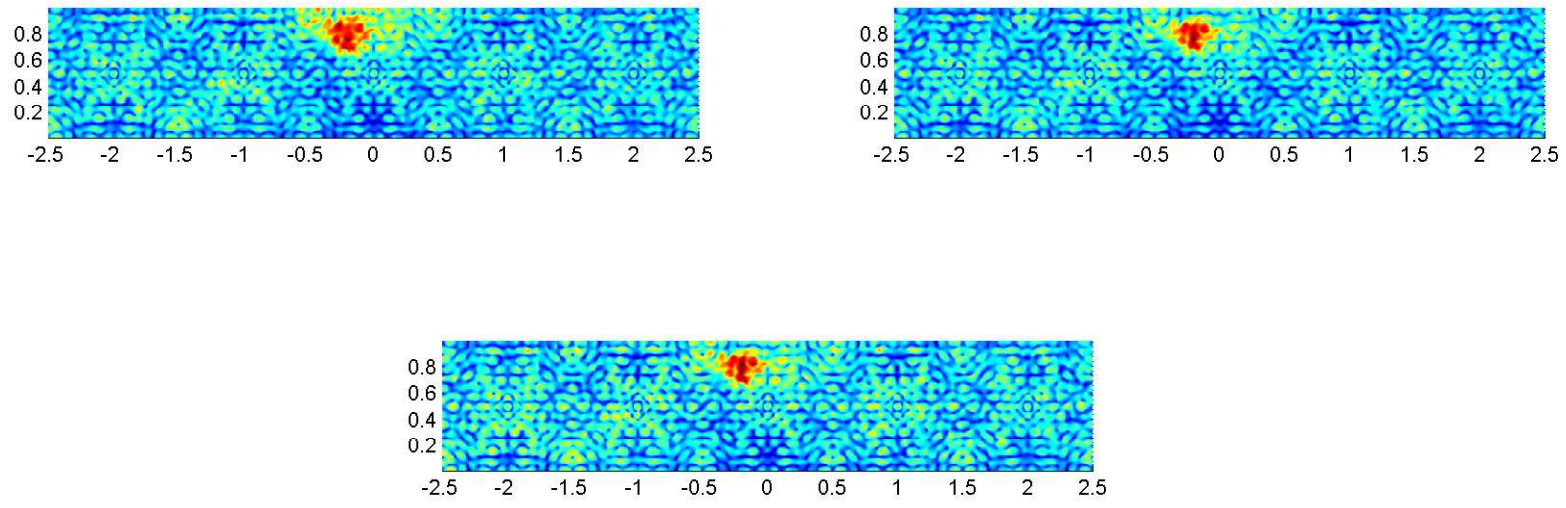

Figure 7. Defect $(i)$. Top left: $\sigma=1 \%$. Top right: $\sigma=5 \%$. Bottom: $\operatorname{defect} \sigma=10 \%$. 


\subsection{The influence of the number of incident waves}

This amounts to analyze the impact of the number $P$ of propagating Floquet modes in each direction of propagation. We consider here the defect $(i i i)$ with $\sigma=1 \%$. It can be seen on figure 8 , on which a comparison between $P=6$ and $P=12$ is done, that having enough propagating Floquet modes used as incident waves is crucial to obtain a good reconstruction.
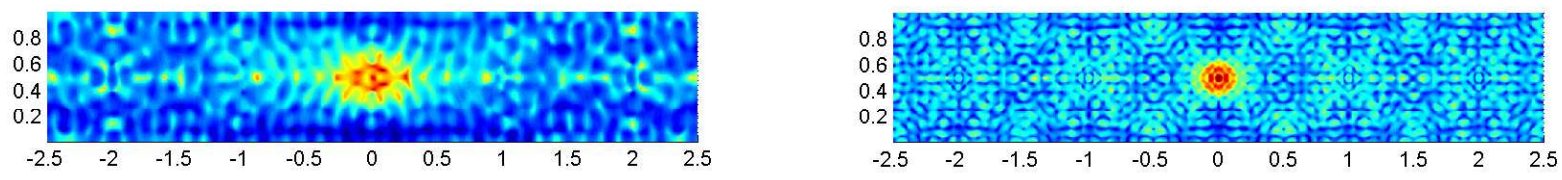

Figure 8. Defect (iii). Left: $P=6$. Right: $P=12$

\subsection{Comparison with the homogeneous waveguide}

We complete this numerical section with a comparison between a periodic waveguide and a homogeneous one. We try to retrieve the same defect, that is a ball centered at $(-0.2,0.8)$ and of radius 0.1 in which $n=2$, on the one hand when the background medium is our periodic waveguide, and on the other hand when the background medium is the homogeneous waveguide characterized by $n_{p}=1$. In order to obtain a meaningful comparison, the number of propagating Floquet modes in the first case has to be equal to the number of propagating guided modes in the second case, here $P=12$. The results are compared in figure 9 for $\sigma=1 \%$ and in figure 10 for $\sigma=5 \%$. We conclude that the identification of defects in a periodic waveguide seems more difficult than in a homogeneous waveguide.
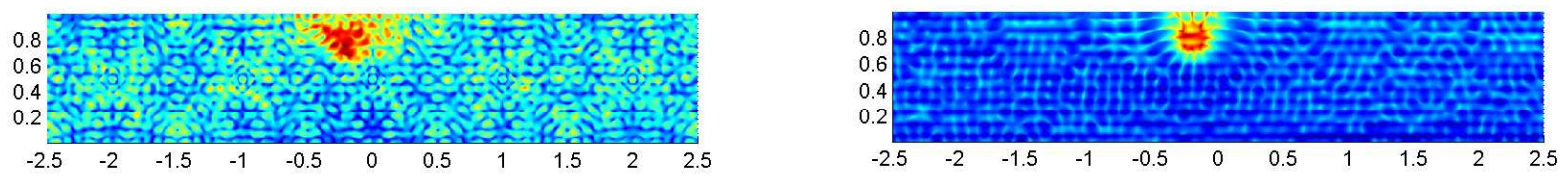

Figure 9. Amplitude of noise: 1\%. Left: periodic waveguide. Right: homogeneous waveguide 

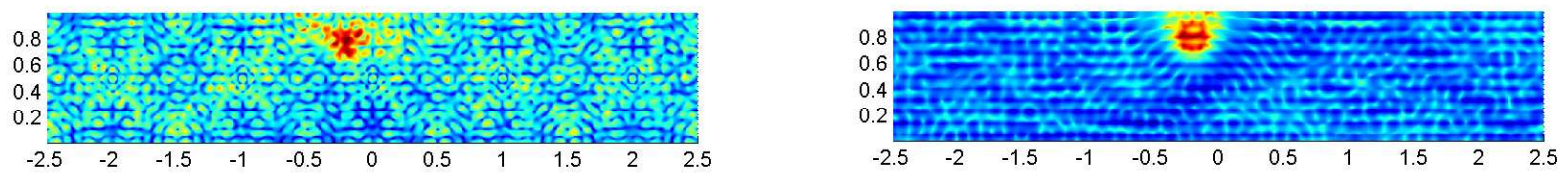

Figure 10. Amplitude of noise: 5\%. Left: periodic waveguide. Right: homogeneous waveguide

\section{Appendix: the homogeneous waveguide as a particular instance of periodic waveguides}

The homogeneous waveguide is obviously a particular case of periodic waveguides for which $n_{p}=1$ in $\Omega$.

Firstly, let us see what are the propagating Floquet modes in that case. For this purpose we introduce the so-called guided modes, which appear when we investigate the solutions of

$$
\left\{\begin{array}{ccc}
-\left(\Delta+k^{2}\right) u=0 & \text { in } & \Omega \\
\partial_{\nu} u=0 & \text { on } & \Gamma .
\end{array}\right.
$$

Indeed, these solutions are the linear combinations of the guided modes given by

$$
u_{m}^{ \pm}\left(x_{1}, x_{2}\right)=\theta_{m}\left(x_{2}\right) e^{ \pm i \beta_{m} x_{1}}, \quad m \geq 1,
$$

with

$$
\begin{aligned}
& \beta_{m}=\sqrt{k^{2}-(m-1)^{2} \pi^{2}}, \quad \operatorname{Re}\left(\beta_{\mathrm{m}}\right), \operatorname{Im}\left(\beta_{m}\right) \geq 0, \\
& \left\{\begin{array}{c}
\theta_{1}\left(x_{2}\right)=1 \\
\theta_{m}\left(x_{2}\right)=\sqrt{2} \cos \left((m-1) \pi x_{2}\right) \quad(m \geq 2) .
\end{array}\right.
\end{aligned}
$$

We assume that $k$ is such that $\beta_{m} \neq 0$ for all $m \geq 1$. Among the guided modes, the propagating guided modes correspond to the purely real $\beta_{m}$, that is $m \in[1, \cdots, P], \quad P:=$ $C(k / \pi)$, where $C$ is the ceiling function. The other ones are either increasing or decreasing exponentially with variable $x_{1}$. Let us verify that the two notions, propagating Floquet modes in the homogeneous case and propagating guided modes, do coincide. It is easy to show that the eigenvalues and eigenfunctions of operator $A(\xi)$ for $n_{p}=1$ are given, for all $m \geq 1$ and $l \in \mathbb{Z}$, by

$$
\mu_{m, l}(\xi)=(m-1)^{2} \pi^{2}+(\xi+2 \pi l)^{2}, \quad \psi_{m, l}(x ; \xi)=\theta_{m}\left(x_{2}\right) e^{i(\xi+2 \pi l) x_{1}} .
$$

Hence the $\lambda_{n}(\xi)$ are obtained from the $\mu_{m, l}(\xi)$ by ordering them for fixed $\xi$. The propagating Floquet modes are then given, for $x \in \Omega$, by

$$
u_{m, l}(x ; \xi)=\theta_{m}\left(x_{2}\right) e^{i(\xi+2 \pi l) x_{1}},
$$


for all $\xi \in(-\pi, \pi], m \geq 1$ and $l \in \mathbb{Z}$ such that

$$
(m-1)^{2} \pi^{2}+(\xi+2 \pi l)^{2}=k^{2} .
$$

Let us consider a propagating Floquet mode $u_{m, l}(\cdot, \xi)$. Since (36) is satisfied, then $\xi+2 \pi l= \pm \sqrt{k^{2}-(m-1)^{2} \pi^{2}}= \pm \beta_{m}$ and therefore $u_{m, l}=u_{m}^{ \pm}$given by (31), that is $u_{m, l}$ is a propagating guided mode. Conversely, let us consider a propagating guided mode $u_{m}^{ \pm}$. There is a unique pair $(\xi, l)$ with $\xi \in(-\pi, \pi]$ and $l \in \mathbb{Z}$ such that $\pm \beta_{m}=\xi+2 \pi l$ (we denote $\left.\xi= \pm \beta_{m} \bmod 2 \pi\right)$. So that $u_{m}^{ \pm}$coincide with the propagating Floquet mode $u_{m, l}(\cdot, \xi)$ given by (35). This completes the verification.

For some index $n \in I(k)$ and $\xi \in \Xi_{n}(k)$, we associate the unique corresponding pair $(m, l)$ such that the propagating Floquet mode $u_{n}(\cdot ; \xi)=u_{m, l}(\cdot ; \xi)$ coincides with a guided mode $u_{m}^{+}$or $u_{m}^{-}$. We have then, for $n \in I(k), \Xi_{n}(k)=\left\{ \pm \beta_{m} \bmod 2 \pi\right\}$ and by using the expression of $\mu_{m, l}$ in (34) we obtain that for $\xi \in \Xi_{n}(k), \lambda_{n}^{\prime}(\xi)= \pm 2 \beta_{m}$.

Remark 6.1. One particular property of periodic waveguides is that there may have band gaps in the spectrum, that is intervals of $k$ for which no propagating Floquet mode exist, in other words $I(k)=\emptyset$. More generally, the number of propagating Floquet modes does not necessarily increase with $k$. This is in contrast with the case of the homogeneous waveguide with Neumann boundary conditions, for which at least two propagating modes $u_{1}^{ \pm}$exist whatever $k$ is, and the number of propagating modes increases with $k$.

Secondly, let us see what becomes the expression (12) of the far field of the fundamental solution when the waveguide is homogeneous. By using the computations above we obtain that such far field is given by

$$
G_{\infty}^{ \pm}(x, y)=i \sum_{m=1}^{P} \frac{u_{m}^{ \pm}(x) u_{m}^{\mp}(y)}{2 \beta_{m}},
$$

where the guided modes $u_{m}^{ \pm}$are given by (31) and the $\beta_{m}$ are given by (32), while $P$ is the number of propagating modes in each direction of propagation. Fortunately, we retrieve such expression by considering directly the far field of the fundamental solution of the homogeneous waveguide, that is:

$$
G(x, y)=i \sum_{m=1}^{+\infty} \frac{e^{i \beta_{m}\left|x_{1}-y_{1}\right|}}{2 \beta_{m}} \theta_{m}\left(x_{2}\right) \theta_{m}\left(y_{2}\right),
$$

where the $\theta_{m}$ are given by (33).

\section{References}

[1] T. Arens, D. Gintides, And A. Lechleiter, Variational formulations for scattering in a threedimensional acoustic waveguide, Math. Methods Appl. Sci., 31 (2008), pp. 821-847.

[2] T. Arens, D. Gintides, And A. Lechleiter, Direct and inverse medium scattering in a threedimensional homogeneous planar waveguide, SIAM Journal on Applied Mathematics, 71 (2011), pp. 753-772. 
[3] T. AREns AND A. KIRsch, The factorization method in inverse scattering from periodic structures, Inverse Problems, 19 (2003), pp. 1195-1211.

[4] L. Bourgeois, F. Le Louer, And E. Lunéville, On the use of lamb modes in the linear sampling method for elastic waveguides, Inverse Problems, 27 (2011), p. 055001.

[5] L. Bourgeois AND E. LunÉville, The linear sampling method in a waveguide: a modal formulation, Inverse Problems, 24 (2008), p. 015018.

[6] - On the use of sampling methods to identify cracks in acoustic waveguides, Inverse Problems, 28 (2012), p. 105011.

[7] - On the use of the linear sampling method to identify cracks in elastic waveguides, Inverse Problems, 29 (2013), p. 025017.

[8] F. CAKoni And D. Colton, Qualitative methods in inverse scattering theory, Interaction of Mechanics and Mathematics, Springer-Verlag, Berlin, 2006. An introduction.

[9] A. Charalambopoulos, D. Gintides, K. Kiriaki, and A. Kirsch, The factorization method for an acoustic wave guide, 7th Int. Workshop on Mathematical Methods in Scattering Theory and Biomedical Engineering, (2006), pp. 120-127.

[10] D. Colton, L. PÄIvärinta, And J. Sylvester, The interior transmission problem, Inverse Probl. Imaging, 1 (2007), pp. 13-28.

[11] D. Colton, M. Piana, and R. Potthast, A simple method using morozov's discrepancy principle for solving inverse scattering problems, Inverse Problems, 13 (1997), pp. 1477-1493.

[12] S. FLiss, Etude mathématique et numérique de la propagation des ondes dans des milieux périodiques localement perturbés, $\mathrm{PhD}$ thesis, 52009.

[13] S. Fliss AND P. Joly, Asymptotic behaviour of solutions of the helmholtz equation in periodic waveguides, In preparation.

[14] T. Hohage And S. Soussi, Riesz bases and jordan form of the translation operator in semi-infinite periodic waveguides, Journal de Mathématiques Pures et Appliquées, 100 (2013), pp. 113 - 135.

[15] P. Joly, J.-R. Li, AND S. Fliss, Exact boundary conditions for periodic waveguides containing a local perturbation, Communications in Computational Physics, 1, 6 (2006), pp. 945-973.

[16] T. Kato, Perturbation theory for linear operators, Springer, 1995.

[17] A. Kirsch And N. GRInBerG, The factorization method for inverse problems, vol. 36 of Oxford Lecture Series in Mathematics and its Applications, Oxford University Press, Oxford, 2008.

[18] A. LeCheiter And D. L. NGuYen, Factorization method for electromagnetic inverse scattering from biperiodic structures, SIAM Journal of Scientific Computing, to appear.

[19] A. LEChLEITER, The factorization method is independent of transmission eigenvalues, Inverse Probl. Imaging, 3 (2009), pp. 123-138.

[20] P. Monk And V. Selgas, Sampling type methods for an inverse waveguide problem, Inverse Problems and Imaging, 6 (2012), pp. 709-747.

[21] R. Potthast, J. Sylvester, And S. Kusiak, A 'range test' for determining scatterers with unknown physical properties, Inverse Problems, 19 (2003), pp. 533-547.

[22] Y. Xu, C. Mawata, And W. Lin, Generalized dual space indicator method for underwater imaging, Inverse Problems, 16 (2000), pp. 1761-1776. 